\title{
Chemical fingerprinting and quantitative constituent analysis of Siwu decoction categorized formulae by UPLC-QTOF/MS/MS and HPLC-DAD
}

Shulan Su' ${ }^{1}$ Wenxia Cui ${ }^{2,1}$, Wei Zhou ${ }^{1}$, Jin-ao Duan ${ }^{1 *}$, Erxin Shang ${ }^{1}$ and Yuping Tang ${ }^{1}$

\begin{abstract}
Background: Siwu decoction categorized formulae (SWDCF) are widely used for treating gynecological diseases. This study aims to elucidate the differences of bioactive constituents in SWDCF by ultra-high performance liquid chromatography coupled with time-of-flight mass spectrometry (UPLC - QTOF - MS /MS) and HPLC-DAD.

Methods: An efficient method based on UPLC - QTOF - MS /MS was developed for identifying the chemical profiles of SWDCF. HPLC-DAD method was used for quantifying seven chemical markers in SWDCF.

Results: Eighty four components were identified or characterized, including ten organic acids, thirty glycosides (monoterpene or iridoid or phenylpropanoids glycosides), fourteen lactones, eighteen flavonoids, and eleven alkaloids in the complex system. The datasets of $t R-m / z$ pairs, ion intensities and sample codes were processed with supervised orthogonal partial least squared discriminant analysis to compare these decoction samples. After a clear classification was established, OPLS-DA was performed and 16 common components with relative quantity in SWDCF samples were determined. Gallic acid, protocatechuic acid, vanillic acid, caffeic acid, paeoniflorin, ferulic acid, and senkyunolide I were selected as the chemical markers to identify SWDCF by HPLC-DAD.
\end{abstract}

Conclusion: The chemical profiles with 84 components in SWDCF, including monoterpene glycosides, acetophenones, galloyl glucoses, even some isomers in the complex system were characterized by UPLC-QTOF-MS/MS.

\section{Background}

Chinese medicine $(\mathrm{CM})$ is holistically formulated for treating complicated CM syndromes ("ZHENG" in Chinese) [1]. The researches on categorized formulae $(\mathrm{CF})$ based on a basic formula composition may help understand the rules of formulation and Fang-Zheng consistency in CM [2].

Siwu decoction (SWD) is a classical prescription that is widely used for the treatment of women's diseases in CM, such as relief of emmeniopathy, climacteric syndrome, dysmenorrhea and other estrogen-related diseases [3]. A recent study demonstrated that the SWD can be synergistically used with Western medicine [4]. SWD consists of four herbs, i.e., Angelicae sinensis Radix, Chuanxiong Rhizoma, Paeoniae Radix Alba, and Rehmanniae Radix

\footnotetext{
* Correspondence: duanja@163.com

1 Jiangsu Key Laboratory for TCM Formulae Research, Nanjing University of Chinese Medicine, Nanjing 210023, PR China

Full list of author information is available at the end of the article
}

[5]. A series of SWD-based formulae used to treat women's diseases especially for primary dysmenorrheal (PD) are considered as Siwu decoction categorized formulae (SWDCF), including Taohong Siwu decoction (THSWD), Xiangfu Siwu decoction (XFSWD), Shaofu Zhuyu decoction (SFZYD), and Qinlian Siwu decoction (QLSWD). The compositions and applications of SWDCF were described in Table 1 . These formulae are usually adopted to treat different symptoms of different kinds of PD.

Our recent studies [6,7] showed that SWDCF inhibited uterine contraction and had analgesic effects on primary dysmenorrhea model mice. Their anti-inflammatory activities, hemorheological improvement and ovarian regulation in rats with blood stasis were elucidated $[8,9]$. Moreover, SWDCF inhibited COX-2 enzyme and platelet aggregation in vitro $[9,10]$. The main constituents of SWDCF belong to several natural product groups, such as phenolic acids, phthalides, alkaloids, terpene glycosides,

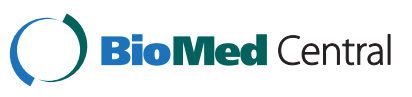


Table 1 The composition and application of SWDCF

\begin{tabular}{|c|c|c|}
\hline Prescriptions & Composition & Application \\
\hline Siwu decoction (SWD) & $\begin{array}{l}\text { (Angelica sinensis) Angelicae sinensis Radix } 9 \mathrm{~g} \text {, (Ligustium chuanxiong) } \\
\text { Chuanxiong Rhizoma } 9 \mathrm{~g} \text {, (Paeonia lactiflora) Paeoniae Radix Alba } 9 \mathrm{~g} \text {, } \\
\text { and (Rehmannia glutinosa) Rehmanniae Radix } 9 \mathrm{~g}\end{array}$ & $\begin{array}{l}\text { Cure women' diseases, such } \\
\text { as dysmenorrhea and other } \\
\text { estrogen related diseases }\end{array}$ \\
\hline Taohong-Siwu decoction (THSWD) & $\begin{array}{l}\text { (Angelica sinensis) Angelicae sinensis Radix } 9 \mathrm{~g} \text {, (Ligustium chuanxiong) } \\
\text { Chuanxiong Rhizoma } 9 \mathrm{~g} \text {, (Paeonia lactiflora) Paeoniae Radix Alba } 9 \mathrm{~g} \text {, } \\
\text { (Rehmannia glutinosa) Rehmanniae Radix } 9 \mathrm{~g} \text {, (Prunus persica) Persicae } \\
\text { Semen } 9 \mathrm{~g} \text {, and (Carthamus tinctorius) Carthami Flos } 6 \mathrm{~g}\end{array}$ & $\begin{array}{l}\text { Cure PD with syndrome of } \\
\text { deficiency of blood. }\end{array}$ \\
\hline Xiangfu-Siwu decoction (XFSWD) & $\begin{array}{l}\text { (Angelica sinensis) Angelicae sinensis Radix } 9 \mathrm{~g} \text {, (Ligustium chuanxiong) } \\
\text { Chuanxiong Rhizoma } 4.5 \mathrm{~g} \text {, (Paeonia lactiflora) Paeoniae Radix Alba } 4.5 \mathrm{~g} \text {, } \\
\text { (Rehmannia glutinosa) Rehmanniae Radix } 12 \mathrm{~g} \text {, (Cyperus rotundus) Cyperi } \\
\text { Rhizoma, } 4.5 \mathrm{~g} \text {, (Corydalis yanhusuo) Corydalis Rhizoma } 4.5 \mathrm{~g} \text {, and } \\
\text { (Aucklandia lappa) Aucklandiae Radix } 3 \mathrm{~g}\end{array}$ & $\begin{array}{l}\text { Cure PD induced by } \\
\text { stagnation of the circulation } \\
\text { of vital energy }\end{array}$ \\
\hline Shaofu-Zhuyu decoction (SFZYD) & $\begin{array}{l}\text { (Angelica sinensis) Angelicae sinensis Radix } 9 \mathrm{~g} \text {, (Ligustium chuanxiong) } \\
\text { Chuanxiong Rhizoma } 3 \mathrm{~g} \text {, (Corydalis yanhusuo)Corydalis Rhizoma } 3 \mathrm{~g} \text {, } \\
\text { (Paeonia veitchii) Paeoniae Radix Rubra } 6 \mathrm{~g} \text {, (Cinnamomum cassia) } \\
\text { Cinnamomi Cortex } 3 \mathrm{~g} \text {, (Foeniculum vulgare) Foeniculi Fructus } 1.5 \mathrm{~g} \text {, } \\
\text { (Zingiber officinale) Zingiberis Rhizoma } 3 \mathrm{~g} \text {, (Commiphora myrrha) Myrrha } \\
3 \mathrm{~g} \text {, (Trogopterprus xanthipes) Trogopterpri Faeces } 6 \mathrm{~g} \text {, and (Typha } \\
\text { angustifolia) Typhae Pollen } 9 \mathrm{~g}\end{array}$ & $\begin{array}{l}\text { Cure PD with syndrome of } \\
\text { cold coagulation and blood } \\
\text { stasis }\end{array}$ \\
\hline Qinlian-Siwu decoction (QLSWD) & $\begin{array}{l}\text { (Angelica sinensis) Angelicae sinensis Radix } 9 \text { g, (Ligustium chuanxiong) } \\
\text { Chuanxiong Rhizoma } 9 \mathrm{~g} \text {, (Paeonia lactiflora) Paeoniae Radix Alba } 9 \mathrm{~g}, \\
\text { and (Rehmannia glutinosa) Rehmanniae Radix } 9 \text { g, (Scutellaria baicalensis) } \\
\text { Scutellariae Radix } 4.5 \mathrm{~g} \text { and (Coptis chinensis) Coptidis Rhizoma } 4.5 \mathrm{~g}\end{array}$ & $\begin{array}{l}\text { Cure PD resulted from } \\
\text { pathogenic heat or } \\
\text { inflammation }\end{array}$ \\
\hline
\end{tabular}

iridoid glycosides, flavones, and etc. [10-13]. However, no report on the constituent profiles of SWDCF is available.

The investigations of the components of SWDCF are important to reveal their effects and action mechanisms. However, the complicated chemical profiles of SWDCF components demand a rapid and efficient method for chemical profiling of SWDCF.

This study aims to profile the constituents of SWDCF by UPLC-QTOF-MS/MS with MarkerLynx analysis and identify SWDCF with several chemical markers.

\section{Methods}

\section{Chemicals, reagents and materials}

Gallic acid, protocatechuic acid, vanillic acid, caffeic acid, paeoniflorin, ferulic acid, and senkyunolide I (Figure 1) were purchased from National Institute for the Control of Pharmaceutical and Biological products (China).

Acetonitrile was HPLC-grade from Merck (Darmstadt, Germany) and deionized water was purified by a Millipore water purification system (Millipore, Milford, MA, USA) and filtered with $0.22 \mu \mathrm{m}$ membranes. Other reagent<smiles>O=C(O)c1cc(O)c(O)c(O)c1</smiles><smiles>O=C(O)c1ccc(O)c(O)c1</smiles>

1. gallic acid<smiles>COc1cc(C(=O)O)ccc1O</smiles>

3. vanillic acid<smiles>O=C(O)/C=C/c1ccc(O)c(O)c1</smiles>

4. caffeic acid

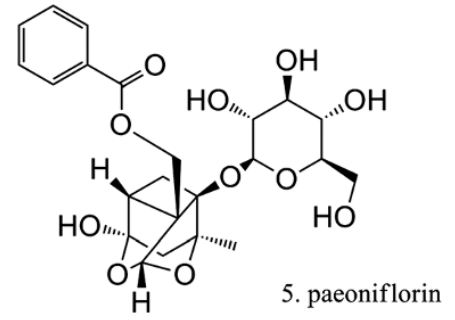<smiles>COc1cc(/C=C/C(=O)O)ccc1O</smiles>

6. ferulic acid<smiles>CCC/C=C1\OC(=O)C2=C1CC[C@@H](O)[C@H]2O</smiles>

7. senkyunolide I

Figure 1 Chemical structures of the quantitative compounds in the Siwu decoction categorized formulae. 
solutions were of analytical grade (Sinopharm Chemical Reagent Co., Ltd., Shanghai, China).

Herbal medicines of Angelicae sinensis Radix, Chuanxiong Rhizoma, Paeoniae Radix Alba, Rehmanniae Radix, Persicae Semen, Carthami Flos, Cyperi Rhizoma, Aucklandiae Radix, Corydalis Rhizoma, Paeoniae Radix Rubra, Cinnamomi Cortex, Foeniculi Fructus, Zingiberis Rhizoma, Myrrha, Trogopterpri Faeces, Typhae Pollen, Scutellariae Radix and Coptidis Rhizoma, were purchased from the following towns (provinces): Minxian (Gansu), Pengzhou (Sichuan), Tongling (Anhui), Huaiqing (Hehan), Anguo (Hebei), Tacheng (Xinjiang), Linyi (Shandong), Lijiang (Yunnan), Songyang (Zhejiang), Chifeng (Neimeng), Yulin (Guangsi), Wuwei (Gansu), Yulin (Guangsi), Guangdong, Changzhi (Shanxi), Yixing (Jiangsu), Chengde (Hebei), and Mianyang (Sichuan), respectively. All crude herbs were identified by the Prof. Jin-ao Duan in accordance with the Pharmacopoeia of People's Republic of China [14]. The voucher specimens (no. NJUTCM-20101112-20101129) were deposited in Jiangsu Key Laboratory for TCM Formulae Research, Nanjing University of Chinese Medicine.

\section{Apparatus and chromatographic conditions UPLC-QTOF-MS/MS qualitative analysis}

Chromatography was performed on an AcQuity ${ }^{\text {TM }}$ UPLC system with a conditioned autosampler (Waters Corp., Milford, MA, USA) at $4^{\circ} \mathrm{C}$. The separation was carried out on an AcQuity UPLCTM BEH $\mathrm{C}_{18}$ column (100 mm $\times$ $2.1 \mathrm{~mm}$ i.d., $1.7 \mu \mathrm{m}$; Waters Corp., Milford, MA, USA) maintained at $35^{\circ} \mathrm{C}$. The mobile phase consisted of $0.1 \%$ formic acid $(\mathrm{HCOOH})$ in water as solvent $\mathrm{A}$ and acetonitrile $(\mathrm{ACN})$ as solvent $\mathrm{B}$. The gradient conditions of the mobile phase were: $0 \mathrm{~min} 95 \% \mathrm{~A}, 9.0 \mathrm{~min} 56 \% \mathrm{~A}$, $12.0 \mathrm{~min} 26 \% \mathrm{~A}, 20.0 \mathrm{~min} 10 \% \mathrm{~A}, 22.0 \mathrm{~min} 10 \% \mathrm{~A}$, $25.0 \mathrm{~min} 95 \% \mathrm{~A}$. The flow rate was $0.40 \mathrm{~mL} / \mathrm{min}$. The sample injection volume was $5 \mu \mathrm{L}$.

Mass spectrometric detection was carried out on an AcQuity Synapt Mass Spectrometer equipped with an electrospray ionization (ESI) interface (Waters, Milford, MA, USA). High purity nitrogen was used as the nebulizer and auxiliary gas; argon was utilized as the collision gas. The ESI source was operated in positive and negative ionization mode with a capillary voltage of $3 \mathrm{kV}$, sampling cone voltage of $10 \mathrm{~V}$, cone gas flow of $50 \mathrm{~L} / \mathrm{h}$, desolvation gas flow of $700 \mathrm{~L} / \mathrm{h}$, desolvation temperature of $350^{\circ} \mathrm{C}$, source temperature of $120^{\circ} \mathrm{C}$, collision energy of $45 \mathrm{~V}$, and the full scan spectra from 100 to 1000 Da. Leucine-enkephalin was used as the lock mass generating an $[\mathrm{M}+\mathrm{H}]^{+}$ion $(\mathrm{m} / z$ $556.2771)$ and $[\mathrm{M}-\mathrm{H}]^{-}$ion $(\mathrm{m} / z 554.2615)$ at a concentration of $200 \mathrm{pg} / \mathrm{mL}$ and flow rate of $100 \mu \mathrm{L} / \mathrm{min}$. Data acquisition and processing were performed by MassLynx 4.1 and MarkerLynx 4.1 (Waters Corp., Milford, MA, USA) for peak detection.

\section{HPLC-PDA quantificative analysis}

The quantification analysis was performed on a Waters2695 Alliance HPLC (Waters Corporation, Milford, MA, USA) equipped with an on-line degasser, an auto-sampler and a 2996 photodiode array detector. UV detection was achieved at $210-400 \mathrm{~nm}$. A Waters Sun Fire ${ }^{\mathrm{TM}} \mathrm{C}_{18}$ column $(4.6 \times 250 \mathrm{~mm}, 5 \mu \mathrm{m}$, serial no. 186002560 Waters Corporation, USA) was used. A linear gradient elution of $\mathrm{A}$ $\mathrm{CH}_{3} \mathrm{OH}$ and $\mathrm{B}\left(\mathrm{CH}_{3} \mathrm{COOH}: \mathrm{H}_{2} \mathrm{O}=0.1\right.$ : 100) was used. The gradient program is $85 \% \mathrm{~B}$ in $0-8 \mathrm{~min}, 85-80 \% \mathrm{~B}$ in $8-10 \mathrm{~min}, 80-74 \% \mathrm{~B}$ in $10-30 \mathrm{~min}, 74-66 \% \mathrm{~B}$ in $30-$ $40 \mathrm{~min}, 66-34 \%$ B in $40-60 \mathrm{~min}, 34-10 \%$ B in $60-85 \mathrm{~min}$. The solvent flow rate was $1 \mathrm{~mL} / \mathrm{min}$ and the column temperature was set at $30^{\circ} \mathrm{C}$. Re-equilibration duration was 15 min between individual runs. A Waters 2996 photo diode array was connected to the liquid chromatography for detection of the raw data.

\section{Preparation of standard solutions}

A mixed standard stock solution containing gallic acid (1), protocatechuic acid (2), vanillic acid (3), caffeic acid (4), paeoniflorin (5), ferulic acid (6), and senkyunolide I (7) was prepared in methanol. The working standard solutions were prepared by diluting the mixed standard solution with methanol to a series of proper concentrations within the ranges: 1, 148-1480.0 $\mu \mathrm{g} / \mathrm{mL} ; 2,38.40-$ $384.00 \mu \mathrm{g} / \mathrm{mL} ; 3$, 32.0-320.0 $\mu \mathrm{g} / \mathrm{mL} ; 4$, 35.6-356.0 $\mu \mathrm{g} / \mathrm{mL} ; \mathbf{5}$, 476.0-4760.0 $\mu \mathrm{g} / \mathrm{mL} ; \quad 6, \quad 136.0-1360.0 \mu \mathrm{g} / \mathrm{mL} ; \quad 7,46.0-$ $460.0 \mu \mathrm{g} / \mathrm{mL}$. The standard stock and working solutions were all stored at $4^{\circ} \mathrm{C}$ and filtered through a $0.22 \mu \mathrm{m}$ membrane prior to injection.

\section{Preparation of sample solutions}

The mixtures of SWDCF (Table 1) were crushed into small pieces and refluxed with 10 times water for $2 \mathrm{~h}$ twice. The filtrates from each decoction were combined and concentrated to $1.0 \mathrm{mg} / \mathrm{mL}$ at $70^{\circ} \mathrm{C}$. The filtrates were added $95 \%$ ethanol until the concentration of ethanol was adjusted to $50 \%$. After centrifugation at $3000 \times g$ for $10 \mathrm{~min}$, the supernatant was stored at $4^{\circ} \mathrm{C}$ and filtered through a $0.22 \mu \mathrm{m}$ membrane filter before the UPLC-QTOF-MS analysis and the HPLC-DAD analysis.

\section{Validation of the HPLC method}

\section{Calibration curves, limits of detection and quantification}

The working standard solutions with at least six different concentrations $(\mathbf{1}, 148-1480.0 \mu \mathrm{g} / \mathrm{mL} ; \mathbf{2}, 38.40$ $384.00 \mu \mathrm{g} / \mathrm{mL} ; 3$, 32.0-320.0 $\mu \mathrm{g} / \mathrm{mL}$; 4, 35.6-356.0 $\mu \mathrm{g} / \mathrm{mL}$; 5, 476.0-4760.0 $\mu \mathrm{g} / \mathrm{mL} ; 6,136.0-1360.0 \mu \mathrm{g} / \mathrm{mL} ; 7$, 46.0$460.0 \mu \mathrm{g} / \mathrm{mL}$ ) were analyzed, and the calibration curves were calculated by linear regression of the double logarithmic plots of the peak area versus the concentration of the reference solution injected. The limits of detection and quantification (LODs and LOQs) under the present 
chromatographic conditions in this study were determined by diluting the standard solution when the signal-to-noise ratios $(\mathrm{S} / \mathrm{N})$ of analytes were about 3 and 10 , respectively. The $\mathrm{S} / \mathrm{N}$ was calculated as the peak height divided by the background noise value.

\section{Precision, repeatability and accuracy}

The intra-day and inter-day variations, which were chosen to determine the precision of the developed method, were investigated by determining the seven analytes in six replicates during a single day and by duplicating the experiments on three consecutive days. Variations of the peak area were taken as the measures of precision and expressed as relative standard deviations (R.S.D.).

Repeatability was confirmed with six independent analytical sample solutions prepared according to the methods describing before and expressed by R.S.D. These SWTCF sample solutions was stored at $4^{\circ} \mathrm{C}$, and injected into the HPLC apparatus at 0, 2, 4, 8, and $12 \mathrm{hr}$, respectively, to evaluate the stability of the solution.

Recovery test was performed by adding accurate amounts of the seven standards into a certain amounts separately to evaluate the accuracy of this method. The spiked samples were then extracted, processed, and quantified in accordance with the methods mentioned above. Six replicates were performed for the test. The average recovery percentage was calculated by the formula: recovery $(\%)=($ observed amount - original amount)/spiked amount $\times 100 \%$.

\section{MarkerLynx $^{\mathrm{TM}}$ analysis}

The data obtained from positive and negative ion mode of all determined samples were analyzed by MarkerLynx v4.1 software (Waters, Manchester, UK) with the help of multivariate statistical analysis, to reveal any potential changed components in SWDCF. The original data were processed using the following parameters: initial retention time of $0 \mathrm{~min}$, final retention time of $25 \mathrm{~min}$, and mass in the range $100-1000 \mathrm{Da}$, with a mass tolerance of $0.02 \mathrm{Da}$, mass window of $0.02 \mathrm{Da}$, retention time window of $0.1 \mathrm{~min}$, noise elimination level 6. For peak integration, peak width at $5 \%$ of the height was $1 \mathrm{~s}$, peak-to -peak baseline noise was automatically calculated, and peak intensity threshold was 10 . No specific mass or adduct was excluded. Isotopic peaks were excluded for analysis. For data analysis, a list of the intensities of the peaks detected was generated using $\mathrm{t} R$ and mass data $(\mathrm{m} / \mathrm{z})$ pairs as the identifier of each peak. An ID was assigned to each of these $\mathrm{t} R-\mathrm{m} / z$ pairs in the order of their UPLC elution for data alignment [15]. The process was repeated for each run. After completion, the correct peak intensity data for each $t R-m / z$ pair of the entire batch of samples were aligned in the final data table. The ions that showed the same $\mathrm{t} R$ (with a tolerance of $0.1 \mathrm{~min}$ ) and $\mathrm{m} / z$ value (with a tolerance of $0.05 \mathrm{Da}$ ) in different samples were considered as the same ion. For those peaks hard to be detected in the sample, the ion intensities were documented as zero in the final data table. Before submitted for multivariate analyses, the ion intensities for each detected peak were normalized against the sum of the peak intensities. The resulting threedimensional data comprising of peak number $(\mathrm{t} R-\mathrm{m} / z$ pair), sample name and ion intensity were analyzed by OPLS-DA with the MarkerLynx software.

\section{Results and discussion}

\section{Identification of SWDCF constituents by UPLC-QTOF-MS/}

MS

The multiple chemical components in the SWDCF samples were identified and characterized by both negative and positive ESI modes. The total current chromatograms at the two modes were shown in Additional file 1: Figures S1-1 and 1-2. Diagnostic fragmentations were then checked by MS/MS to confirm the results. Eighty-four constituents were identified by comparing the $\mathrm{t} R, \mathrm{UV}_{\lambda \max }$, and $\mathrm{MS}$ fragments characteristics of the compounds. The analyzed and identified compounds were listed in Table 2. Among these 84 compounds, there were ten organic acids, thirty glycosides (monoterpene or iridoid glycosides or phenylpropanoids), fourteen lactones, eighteen flavonoids, and eleven alkaloids.

\section{Identification of phenolic acids}

In this study, 10 phenolic acids were identified from SWDCF samples according to the $\mathrm{t} R, \mathrm{UV}_{\lambda \max }$, and MS fragment characteristics compared with reference compounds and the literature [16,17]. They are gallic acid (1), chlorogenic acid (2), caffeic acid (3), vanillic acid (4), ferulic acid (5), isoferulic acid (6), protocatechuic acid (7), coumaric acid (8), p-hydroxy benzoic acid (9), and benzoic acid (10), respectively. The MS characteristics (Table 2) were $m / z 169[\mathrm{M}-\mathrm{H}]^{-}, m / z 353[\mathrm{M}-\mathrm{H}]^{-}, m / z 179$ $[\mathrm{M}-\mathrm{H}]^{-}, m / z 167[\mathrm{M}-\mathrm{H}]^{-}, m / z 193[\mathrm{M}-\mathrm{H}]^{-}, m / z 193[\mathrm{M}-$ $\mathrm{H}]^{-}, m / z 153[\mathrm{M}-\mathrm{H}]^{-}, m / z 163[\mathrm{M}-\mathrm{H}]^{-}, m / z 137[\mathrm{M}-\mathrm{H}]^{-}$, and $m / z 121[\mathrm{M}-\mathrm{H}]^{-}$, respectively.

\section{Identification of monoterpene, iridoid and phenylpropanoid glycosides}

In the positive and negative MS experiments, there were $\mathbf{1 6}$ monoterpenes compounds with a pinane skeleton were analyzed and identified (Table 2). These constituents come from herbs of Paeoniae Radix Alba (in SWD, THSWD, XFSWD, and QLSWD) and Paeoniae Radix Rubra (in SFZYD). According to the MS/MS analysis of authentic compounds, the major fragmentation mechanisms of monoterpene glycosides were concluded. In the positive MS experiments, all monoterpene glycosides were ionized as sodiated molecules. The diagnostic ions of this type of compounds were the loss of glucosyl group, aglycone 
Table 2 Characterization of compounds in Siwu decoction categorized formulae by UPLC-QTOF-MS/MS

\begin{tabular}{|c|c|c|c|c|c|c|}
\hline $\begin{array}{l}\text { Peak } \\
\text { no. }\end{array}$ & $T_{R}(\min )$ & Positive ions $(\mathrm{m} / \mathrm{z})$ & Negative ions $(m / z)$ & $\lambda_{\max }(\mathrm{nm})$ & Identification & Origins \\
\hline 1 & 1.42 & $171[\mathrm{M}+\mathrm{H}]^{+}$ & $169[\mathrm{M}-\mathrm{H}]^{-}, 125[\mathrm{M}-\mathrm{HCOO}]^{-}, 97\left[\mathrm{M}-\mathrm{H}-\mathrm{CO}_{2}-\mathrm{CO}\right]^{-}$ & 216,270 & Gallic acid & $A, B, C, D, E$ \\
\hline 2 & 3.07 & $355[\mathrm{M}+\mathrm{H}]^{+}, 377[\mathrm{M}+\mathrm{Na}]^{+}$ & $353[\mathrm{M}-\mathrm{H}]^{-}, 191\left[\mathrm{M}-\mathrm{C}_{9} \mathrm{H}_{7} \mathrm{O}_{3}\right]^{-}, 179\left[\mathrm{M}-\mathrm{C}_{7} \mathrm{H}_{11} \mathrm{O}_{5}\right]^{-}, 173\left[\mathrm{M}-\mathrm{C}_{9} \mathrm{H}_{7} \mathrm{O}_{3}-\mathrm{H}_{2} \mathrm{O}\right]^{-}$ & 326 & Chlorogenic acid & $A, B, C, D, E$ \\
\hline 3 & 4.10 & $181[\mathrm{M}+\mathrm{H}]^{+}$ & $179[\mathrm{M}-\mathrm{H}]^{-}, 135[\mathrm{M}-\mathrm{HCOO}]^{-}$ & 320,240 & Caffeic acid & $A, B, C, D, E$ \\
\hline 4 & 3.52 & $169[\mathrm{M}+\mathrm{H}]^{+}$ & $167[\mathrm{M}-\mathrm{H}]^{-}, 153\left[\mathrm{M}-\mathrm{CH}_{3}\right]^{-}, 137\left[\mathrm{M}-\mathrm{OCH}_{3}\right]^{-}, 123[\mathrm{M}-\mathrm{HCOO}]^{-}$ & 290 & Vanillic acid & $A, B, C, D, E$ \\
\hline 5 & 4.90 & $195[\mathrm{M}+\mathrm{H}]^{+}$ & $193[\mathrm{M}-\mathrm{H}]^{-}, 178\left[\mathrm{M}-\mathrm{H}-\mathrm{CH}_{3}\right]^{-}, 149\left[\mathrm{M}-\mathrm{H}-\mathrm{CO}_{2}\right]^{-}, 134[\mathrm{M}-\mathrm{HCOO}]^{-}$ & 320 & Ferulic acid & $A, B, C, D, E$ \\
\hline 6 & 8.90 & $195[\mathrm{M}+\mathrm{H}]^{+}$ & $193[\mathrm{M}-\mathrm{H}]^{-}, 237[\mathrm{M}-\mathrm{H}+\mathrm{HCOO}]^{-}, 179\left[\mathrm{M}-\mathrm{CH}_{3}\right]^{-}$ & 310 & Isoferulic acid & $A, B, C, D, E$ \\
\hline 7 & 4.68 & - & $153[\mathrm{M}-\mathrm{H}]^{-}, 141\left[\mathrm{M}-\mathrm{H}-\mathrm{CH}_{3}\right]^{-}, 109\left[\mathrm{M}-\mathrm{H}-\mathrm{CO}_{2}\right]^{-}$ & 310 & Protocatechuic acid & $A, B, C, D, E$ \\
\hline 8 & 9.44 & - & $163[\mathrm{M}-\mathrm{H}]^{-}, 117[\mathrm{M}-\mathrm{H}-\mathrm{HCOO}]^{-}$ & & Coumaric acid & $A, B$ \\
\hline 9 & 2.98 & $139[\mathrm{M}+\mathrm{H}]^{+}, 161[\mathrm{M}+\mathrm{Na}]^{+}$ & $137[\mathrm{M}-\mathrm{H}]^{-}, 119\left[\mathrm{M}-\mathrm{H}-\mathrm{H}_{2} \mathrm{O}\right]^{-}$ & & p-hydroxy benzoic acid & $A, B$ \\
\hline 10 & 7.02 & - & $121[\mathrm{M}-\mathrm{H}]^{-}$ & 238,272 & Benzoic acid & $A, B$ \\
\hline 11 & 1.47 & $361[M+H]^{+}$ & $\begin{array}{l}359[\mathrm{M}-\mathrm{H}]^{-} ; 493\left[\mathrm{M}-\mathrm{H}+\mathrm{HCOO}^{-}, 405[\mathrm{M}+\mathrm{HCOO}]^{-}, 197[\mathrm{M}-\mathrm{H}-\mathrm{glu}]^{-}, 179[\mathrm{M}-\right. \\
\left.\mathrm{H}-\mathrm{glu}-\mathrm{H}_{2} \mathrm{O}\right]^{-}\end{array}$ & 210,270 & $\begin{array}{l}\text { 1-O- } \beta \text {-D- glucopyranosyl - } \\
\text { paeonisuffrone }\end{array}$ & $A, B$ \\
\hline 12 & 1.51 & $517[\mathrm{M}+\mathrm{Na}]^{+}$ & $\begin{array}{l}493[\mathrm{M}-\mathrm{H}]^{-}, 457\left[\mathrm{M}-\mathrm{H}-2 \mathrm{H}_{2} \mathrm{O}\right]^{-}, 443\left[\mathrm{M}-\mathrm{H}-2 \mathrm{H}_{2} \mathrm{O}-\mathrm{CH}_{3}\right]^{-}, 331[\mathrm{M}-2 \mathrm{H}-\mathrm{glu}]^{-}, 169 \\
{[\mathrm{M} \text {-sucrose }]^{-}}\end{array}$ & 220,270 & 1'-O-galloylsucrose & A \\
\hline 13 & 2.89 & $\begin{array}{l}562[\mathrm{M}+\mathrm{Na}]^{+}, 383[\mathrm{M}+\mathrm{H}-\mathrm{glu}]^{+} \\
261[\mathrm{M}+\mathrm{H} \text {-glu-benzoyl }]^{+}, 197[\mathrm{M} \\
\left.\left.+\mathrm{H}^{-g l u} \text {-benzoyl-SO }\right]_{2}\right]^{+}\end{array}$ & $\begin{array}{l}543[\mathrm{M}-\mathrm{H}]^{-}, 495\left[\mathrm{M}-\mathrm{CH}_{2} \mathrm{OH}-\mathrm{H}_{2} \mathrm{O}\right]^{-}, 461\left[\mathrm{M}-\mathrm{SO}_{2}-\mathrm{H}_{2} \mathrm{O}-\mathrm{H}^{-}, 341\left[{\mathrm{M}-\mathrm{SO}_{2}-\mathrm{C}_{7} \mathrm{H}_{5} \mathrm{O}-}^{2 \mathrm{OH}]^{-}, 243\left[\mathrm{M}-\text { glu-benzoyl- } \mathrm{H}_{2} \mathrm{O}-\mathrm{CH}_{3}\right]^{-}}\right.\right.\end{array}$ & 232 & Paeoniflorin sulfonate & $A, B$ \\
\hline 14 & 3.85 & $\begin{array}{l}481\left[\mathrm{M}+\mathrm{H}^{+}, 319[\mathrm{M}+\mathrm{H}-\mathrm{glu}]^{+}\right. \\
197[\mathrm{M}+\mathrm{H} \text {-glu-benzoyl }]^{+}, 161 \\
{\left[\mathrm{M}+\mathrm{H} \text {-glu-benzoyl-2 } \mathrm{H}_{2} \mathrm{O}\right]^{+}, 133} \\
{\left[\mathrm{M}+\mathrm{H} \text {-glu-benzoyl- } 2 \mathrm{H}_{2} \mathrm{O}-\mathrm{CO}\right]^{+}}\end{array}$ & $\begin{array}{l}479[\mathrm{M}-\mathrm{H}]^{-}, 525[\mathrm{M}+\mathrm{HCOO}]^{-}, 416\left[\mathrm{M}-\mathrm{CH}_{2} \mathrm{OH}-\mathrm{CH}_{3}-\mathrm{H}_{2} \mathrm{O}\right]^{-}, 283\left[\mathrm{M}-\text { glu- } \mathrm{H}_{2} \mathrm{O}-\right. \\
\mathrm{OH}]^{-}, 177\left[\mathrm{M} \text {-glu-benzoyl-2H }{ }_{2}\right]^{-}\end{array}$ & 233 & Albiflorin & $A, B, C, D, E$ \\
\hline 15 & 4.29 & - & 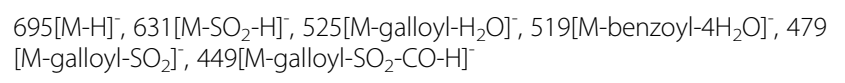 & 275 & $\begin{array}{l}\text { Galloylpaeoniflorin } \\
\text { sulfonate }\end{array}$ & $A, B$ \\
\hline 16 & 5.84 & $\begin{array}{l}481[\mathrm{M}+\mathrm{H}]^{+}, 503[\mathrm{M}+\mathrm{Na}]^{+}, 397 \\
319,197\end{array}$ & 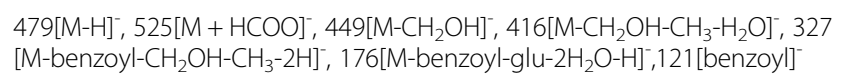 & 230 & Paeoniflorin & $A, B, C, D, E$ \\
\hline 17 & 4.36 & $\begin{array}{l}497\left[\mathrm{M}+\mathrm{H}^{+}, 519[\mathrm{M}+\mathrm{Na}]^{+}, 381\right. \\
{[\mathrm{M}+\mathrm{Na}-\mathrm{pOHBA}]^{+}, 357[\mathrm{M}+\mathrm{Na}-} \\
\mathrm{Glc}^{+}, 323[\mathrm{M}+\mathrm{Na}-\mathrm{aglycone}]^{+} \\
271\left[\mathrm{M}+\mathrm{Na}-\mathrm{C}_{13} \mathrm{H}_{12} \mathrm{O}_{5}\right]^{+}\end{array}$ & $\begin{array}{l}495[\mathrm{M}-\mathrm{H}]^{-}, 449\left[\mathrm{M}-\mathrm{CH}_{3}-\mathrm{CH}_{2} \mathrm{OH}-\mathrm{H}\right]^{-}, 479[\mathrm{M}-\mathrm{OH}]^{-}, 327[\mathrm{M}-\mathrm{p}-\text { hydroxybenzoyl- } \\
\left.\mathrm{CH}_{2} \mathrm{OH}-\mathrm{OH}\right]^{-}\end{array}$ & 235,322 & Oxypaeoniflorin & A \\
\hline 18 & 3.91 & $481[\mathrm{M}+\mathrm{H}]^{+}, 503[\mathrm{M}+\mathrm{Na}]^{+}$ & 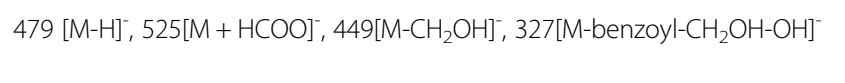 & 220,275 & $\begin{array}{l}\text { Isopaeoniflorin / albiflorin } \\
\text { R1 }\end{array}$ & A \\
\hline 19 & 4.97 & $\begin{array}{l}633\left[\mathrm{M}+\mathrm{H}^{+}, 655[\mathrm{M}+\mathrm{Na}]^{+}, 153\right. \\
{\left[\text { galloyl }+\mathrm{H}-\mathrm{H}_{2} \mathrm{O}\right]^{+}}\end{array}$ & $631[\mathrm{M}-\mathrm{H}]^{-}, 525[\mathrm{M}-\text { benzoyl-2H]', 449[M-galloy-HCHO}]^{-}$ & 275 & Galloylpaeoniflorin & $A, B$ \\
\hline 20 & 4.87 & - & $939[\mathrm{M}-\mathrm{H}]^{-}, 631$ [M-2galloyl-3H]', 469 [M-2galloyl-3H-glu] $]^{-}$ & 220,280 & Pentagalloylglucose & $A, B, C$ \\
\hline 21 & 5.20 & - & $623[\mathrm{M}-\mathrm{H}]^{-}, 515\left[\mathrm{M}-\mathrm{C}_{6} \mathrm{H}_{5} \mathrm{O}_{2}\right]^{-}$ & & $\begin{array}{l}\text { Acteoside/isoacteoside / } \\
\text { forsythoside A }\end{array}$ & $A, B$ \\
\hline
\end{tabular}


Table 2 Characterization of compounds in Siwu decoction categorized formulae by UPLC-QTOF-MS/MS (Continued)

\begin{tabular}{|c|c|c|c|c|c|c|}
\hline 22 & 5.78 & 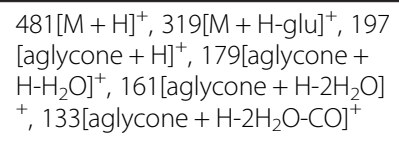 & $479[\mathrm{M}-\mathrm{H}]^{-}, 463[\mathrm{M}-\mathrm{OH}]^{-}, 341[\mathrm{M}$-benzoyl-2OH] & & Mudanpioside I & $A, B$ \\
\hline 23 & 6.67 & & $\begin{array}{l}\left.\text { 647[M-H]', 513[M-benzoyl-HCHO]', 507[M-benzoyl-2 } \mathrm{H}_{2} \mathrm{O}\right]^{-}, 391[\mathrm{M}-2 \text { benzoyl- } \\
\mathrm{HCHO}-\mathrm{OH}]^{-}\end{array}$ & & $\begin{array}{l}\text { Benzoypaeoniflorin } \\
\text { sulfonate }\end{array}$ & $A, B$ \\
\hline 24 & 9.29 & 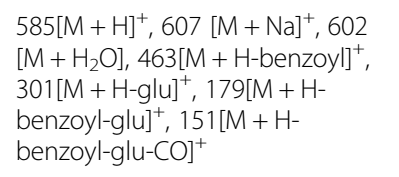 & $583[\mathrm{M}-\mathrm{H}]^{-}, 629[\mathrm{M}+\mathrm{HCOO}]^{-}$ & 220,270 & Benzoylpaeoniflorin & $A, C$ \\
\hline 25 & 8.09 & & $583[\mathrm{M}-\mathrm{H}]^{-}, 629\left[\mathrm{M}+\mathrm{HCOO}^{-}\right.$ & 220,270 & Isobenzoylpaeoniflorin & $A, B$ \\
\hline 26 & 4.20 & - & 799[M-H]', 637[M-caffeoyl]', 525, 479, 449 & & $\begin{array}{l}\text { cistanoside A or jionoside } \\
\text { A1/A2 }\end{array}$ & A \\
\hline 27 & 3.66 & $\begin{array}{l}660\left[\mathrm{M}+\mathrm{H}_{2} \mathrm{O}\right], 625\left[\mathrm{M}+\mathrm{H}-\mathrm{H}_{2} \mathrm{O}\right]^{+} \\
341\left[\mathrm{M}+\mathrm{H}-\mathrm{H}_{2} \mathrm{O}-\mathrm{glu} \text {-benzoyl }\right]^{+} \\
301\left[\mathrm{M}+\mathrm{H}-\mathrm{H}_{2} \mathrm{O}-2 \mathrm{glu}\right]^{+}\end{array}$ & 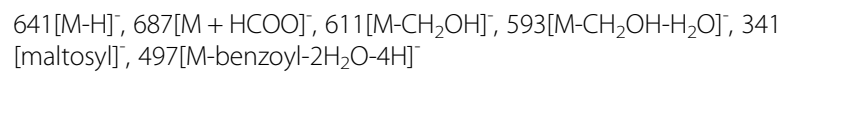 & 212,277 & $\begin{array}{l}\text { Isomaltopaeoniflorin/6'-O- } \\
\beta \text {-D- } \\
\text { glucopyranosylalbiflorin }\end{array}$ & A \\
\hline 28 & 2.75 & $729[\mathrm{M}+\mathrm{Na}]^{+}$ & $705[\mathrm{M}-\mathrm{H}]^{-}, 495,443,341,271,193$ & 215 & $\begin{array}{l}\text { Isomaltopaeoniflorin } \\
\text { sulfonate }\end{array}$ & A \\
\hline 29 & 4.14 & $\begin{array}{l}463\left[\mathrm{M}+\mathrm{H}^{+}, 484[\mathrm{M}+\mathrm{Na}]^{+}, 498\right. \\
301,179\end{array}$ & $461[\mathrm{M}-\mathrm{H}]^{-}, 525,479,449,327$ & 232 & decaffeoyl-verbascoside & A \\
\hline 30 & 5.68 & $\begin{array}{l}363\left[\mathrm{M}+\mathrm{H}^{+}, 385[\mathrm{M}+\mathrm{Na}]^{+}, 340\right. \\
319,197\end{array}$ & $361[\mathrm{M}-\mathrm{H}]^{-}, 407\left[\mathrm{M}+\mathrm{HCOO}^{-}, 311,287\right.$ & 232 & $\begin{array}{l}\text { 6-O- } \beta \text {-D-glucopyranosyl } \\
\text { lactinolide }\end{array}$ & A \\
\hline 31 & 1.40 & $687[\mathrm{M}+\mathrm{H}]^{+}$ & $685[\mathrm{M}-\mathrm{H}]^{-}, 731\left[\mathrm{M}-\mathrm{H}+\mathrm{HCOO}^{-}, 493\left[\mathrm{M}-\mathrm{H}-\mathrm{glu}-\mathrm{HCOO}^{-}\right.\right.$ & 215,273 & Rehmannioside D & $A, B$ \\
\hline 32 & 7.18 & $525[\mathrm{M}+\mathrm{H}]^{+}, 207$ & $523[\mathrm{M}-\mathrm{H}]^{-}, 569[\mathrm{M}+\mathrm{HCOO}]^{-}, 407,341,183$ & & Melittoside & $A, B$ \\
\hline 33 & 3.71 & - & $785[\mathrm{M}-\mathrm{H}]^{-}, 687[\mathrm{M}-98]^{-}$ & 236,279 & Echinacoside & A \\
\hline 34 & 4.55 & & $435[\mathrm{M}+\mathrm{HCOO}]^{-}, 389[\mathrm{M}-\mathrm{H}]^{-}, 327\left[\mathrm{M}-3 \mathrm{CH}_{3}-\mathrm{H}_{2} \mathrm{O}\right]^{-}, 178\left[\mathrm{M}-\mathrm{C}_{13} \mathrm{H}_{24} \mathrm{O}_{2}\right]^{-}$ & - & Rehmaionoside A/B & $A, B$ \\
\hline 35 & 4.94 & - & $813[\mathrm{M}-\mathrm{H}]^{-}, 515\left[\mathrm{M}-\mathrm{C}_{6} \mathrm{H}_{12} \mathrm{O}_{4}-\mathrm{C}_{9} \mathrm{H}_{10} \mathrm{O}_{2}-\mathrm{H}\right]^{-}, 469[\mathrm{M}-\text { glu-feruloyl] }]^{-}$ & & Jionoside B1/B2 & $A, B$ \\
\hline 36 & 6.51 & $653[\mathrm{M}+\mathrm{H}]^{+}$ & $651[\mathrm{M}-\mathrm{H}]^{-}, 505[\mathrm{M}-\text { rhamnosyl-H] }]^{-}$ & 276 & Martynoside isomer & A \\
\hline 37 & 4.98 & $653[\mathrm{M}+\mathrm{H}]^{+}$ & $651[\mathrm{M}-\mathrm{H}]^{-}, 445\left[\mathrm{M} \text {-ferulic acid-2 } \mathrm{CH}_{3}\right]^{-}, 389\left[\mathrm{M}\right.$-feruloyl- $\mathrm{HCHO}-3 \mathrm{H}_{2} \mathrm{O}-2 \mathrm{H}^{-}$ & 235,322 & Martynoside & A \\
\hline 38 & 5.52 & $347[\mathrm{M}+\mathrm{H}]^{+}$ & 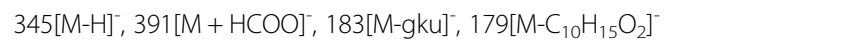 & - & Rehmapicroside & $A, E$ \\
\hline 39 & 7.21 & $207[\mathrm{M}+\mathrm{Na}]^{+}$ & $183[\mathrm{M}-\mathrm{H}]^{-}, 165\left[\mathrm{M}-\mathrm{H}_{2} \mathrm{O}-\mathrm{H}\right]^{-}, 139[\mathrm{M}-\mathrm{HCOO}]^{-}$ & - & Rehmapicrogenin & $A, E$ \\
\hline 40 & 3.20 & $459[\mathrm{M}+\mathrm{H}]^{+}, 325,163$ & $457[\mathrm{M}-\mathrm{H}]]^{-}, 323$ & 215,237 & Amygdalin & B \\
\hline 41 & 6.09 & $\begin{array}{l}249[\mathrm{M}+\mathrm{Na}]^{+}, 227\left[\mathrm{M}+\mathrm{H}^{+}, 209\right. \\
{\left[\mathrm{M}-\mathrm{H}_{2} \mathrm{O}+\mathrm{H}^{+}, 191\left[\mathrm{M}-2 \mathrm{H}_{2} \mathrm{O}+\mathrm{H}^{+}\right.\right.} \\
181\left[\mathrm{M}-\mathrm{H}_{2} \mathrm{O}-\mathrm{CO}+\mathrm{H}\right]^{+}\end{array}$ & $225[\mathrm{M}-\mathrm{H}], 195,125$ & 277 & Senkyunolide J & $A, B, C, D, E$ \\
\hline 42 & 6.26 & $\begin{array}{l}225[\mathrm{M}+\mathrm{H}]^{+}, 247[\mathrm{M}+\mathrm{Na}]^{+} \\
207.1014\left[\mathrm{M}-\mathrm{H}_{2} \mathrm{O}+\mathrm{H}\right]^{+}, 189.0889 \\
{\left[\mathrm{M}+\mathrm{H}-2 \mathrm{H}_{2} \mathrm{O}\right]^{+}, 165.0890[\mathrm{M}+\mathrm{H}-} \\
\left.\mathrm{H}_{2} \mathrm{O}-\mathrm{C}_{3} \mathrm{H}_{6}\right]^{+}\end{array}$ & 223[M-H]', 205, 177 & 270 & Senkyunolide I & $A, B, C, D, E$ \\
\hline
\end{tabular}

$641[\mathrm{M}-\mathrm{H}]^{\prime}, 687[\mathrm{M}+\mathrm{HCOO}]^{\prime}, 611\left[\mathrm{M}-\mathrm{CH}_{2} \mathrm{OH}\right]^{-}, 593\left[\mathrm{M}-\mathrm{CH}_{2} \mathrm{OH}-\mathrm{H}_{2} \mathrm{O}\right]^{-}, 341$

705[M-H]', 495, 443, 341, 271, 193

215,273

236,279

$435[\mathrm{M}+\mathrm{HCOO}]^{-}, 389[\mathrm{M}-\mathrm{H}]^{-}, 327\left[\mathrm{M}-3 \mathrm{CH}_{3}-\mathrm{H}_{2} \mathrm{O}\right]^{-}, 178\left[\mathrm{M}-\mathrm{C}_{13} \mathrm{H}_{24} \mathrm{O}_{2}\right]$

$651[\mathrm{M}-\mathrm{H}]$ ] , 445[M-ferulic acid-2CH$]_{3}$ ], 389[M-feruloyl-HCHO-3H $\mathrm{H}_{2} \mathrm{O}-2 \mathrm{H}$ ]

$457[\mathrm{M}-\mathrm{H}], 323$

215,237

$249[\mathrm{M}+\mathrm{Na}]^{+}, 227\left[\mathrm{M}+\mathrm{H}^{+}, 209\right.$ $\mathrm{M}-\mathrm{H}_{2} \mathrm{O}+\mathrm{H}^{+}, 191\left[\mathrm{M}-2 \mathrm{H}_{2} \mathrm{O}+\mathrm{H}^{+}\right.$

$225[\mathrm{M}+\mathrm{H}]^{+}, 247[\mathrm{M}+\mathrm{Na}]^{+}$

$\left.\mathrm{M}+\mathrm{H}-2 \mathrm{H}_{2} \mathrm{O}\right]^{+}, 165.0890[\mathrm{M}+\mathrm{H}-$ $\left.\mathrm{H}_{2} \mathrm{O}-\mathrm{C}_{3} \mathrm{H}_{6}\right]^{+}$ 
Table 2 Characterization of compounds in Siwu decoction categorized formulae by UPLC-QTOF-MS/MS (Continued)

\begin{tabular}{|c|c|c|c|c|c|c|}
\hline$\overline{43}$ & 6.61 & $\begin{array}{l}225\left[\mathrm{M}+\mathrm{H}^{+}, 247[\mathrm{M}+\mathrm{Na}]^{+}, 207\right. \\
{\left[\mathrm{M}-\mathrm{H}_{2} \mathrm{O}+\mathrm{H}^{+}, 189\left[\mathrm{M}+\mathrm{H}-2 \mathrm{H}_{2} \mathrm{O}\right]^{+}\right.} \\
165\left[\mathrm{M}+\mathrm{H}-\mathrm{H}_{2} \mathrm{O}-\mathrm{C}_{3} \mathrm{H}_{6}\right]^{+}\end{array}$ & $223[\mathrm{M}-\mathrm{H}]^{-}$ & 270 & Senkyunolide $\mathrm{H}$ & $\overline{A, B, C, D, E}$ \\
\hline 44 & 10.82 & $191[\mathrm{M}+\mathrm{H}]^{+}, 173,149,135$ & $189[\mathrm{M}-\mathrm{H}]^{-}, 207\left[\mathrm{M}-\mathrm{H}+\mathrm{H}_{2} \mathrm{O}\right]^{-}$ & 295,325 & E-ligustilide & $A, B, C, D, E$ \\
\hline 45 & 13.74 & $191[\mathrm{M}+\mathrm{H}]^{+}, 173,149,135$ & - & 295,325 & Z-ligustilide & $A, B, C, D, E$ \\
\hline 46 & 9.44 & $\begin{array}{l}381[\mathrm{M}+\mathrm{H}]^{+}, 426[\mathrm{M}+2 \mathrm{Na}]^{+}, 191 \\
{\left[\mathrm{C}_{12} \mathrm{H}_{15} \mathrm{O}_{2}\right]^{+}}\end{array}$ & - & 280 & Z-ligustilide dimmer E-232 & $A, B, C, D, E$ \\
\hline 47 & 13.62 & $\begin{array}{l}381\left[\mathrm{M}+\mathrm{H}^{+}\right]^{+}, 426[\mathrm{M}+2 \mathrm{Na}]^{+}, 191 \\
{\left[\mathrm{C}_{12} \mathrm{H}_{15} \mathrm{O}_{2}\right]^{+}}\end{array}$ & - & 296 & $z, Z^{\prime}-3,3^{\prime}, 8,8^{\prime}$-Diligustilide & $A, B, C, D$ \\
\hline 48 & 10.33 & $203[\mathrm{M}+\mathrm{H}]^{+}, 225[\mathrm{M}+\mathrm{Na}]^{+}$ & - & - & $\begin{array}{l}\text { 3-butylidene-7- } \\
\text { hydroxyphthalide }\end{array}$ & $A, B, C, D, E$ \\
\hline 49 & 8.04 & $\begin{array}{l}189\left[\mathrm{M}+\mathrm{H}^{+}, 171\left[\mathrm{M}-\mathrm{H}_{2} \mathrm{O}+\mathrm{H}\right]^{+}\right. \\
153\left[\mathrm{M}-2 \mathrm{H}_{2} \mathrm{O}+\mathrm{H}\right]^{+}, 117[\mathrm{M}+3 \mathrm{H}- \\
\left.\mathrm{H}_{2} \mathrm{O}-\mathrm{CO}-\mathrm{C}_{2} \mathrm{H}_{4}\right]^{+}\end{array}$ & $187[\mathrm{M}-\mathrm{H}]^{-}$ & 260,310 & E-Butylideniphthalide & $A, B, C, D, E$ \\
\hline 50 & 9.31 & $\begin{array}{l}189\left[\mathrm{M}+\mathrm{H}^{+}, 161[\mathrm{M}-\mathrm{CO}+\mathrm{H}]^{+}, 133\right. \\
{\left[\mathrm{M}+\mathrm{H}-\mathrm{CO}-\mathrm{C}_{2} \mathrm{H}_{4}\right]^{+}}\end{array}$ & $187[\mathrm{M}-\mathrm{H}]^{-}, 205\left[\mathrm{M}-\mathrm{H}+\mathrm{H}_{2} \mathrm{O}\right]^{-}$ & 260,310 & Z-Butylidenephthalide & $A, B, C, D, E$ \\
\hline 51 & 12.90 & $\begin{array}{l}381\left[\mathrm{M}+\mathrm{H}^{+}, 426[\mathrm{M}+\mathrm{H}+\mathrm{HCOO}]\right. \\
+, 236,191\end{array}$ & $379[\mathrm{M}-\mathrm{H}]^{-}, 424[\mathrm{M}-\mathrm{H}+\mathrm{HCOO}]^{\prime}, 397\left[\mathrm{M}-\mathrm{H}+\mathrm{H}_{2} \mathrm{O}\right]^{-}$ & 282 & Angelicide & A \\
\hline 52 & 13.65 & $\begin{array}{l}381[\mathrm{M}+\mathrm{H}]^{+}, 403[\mathrm{M}+\mathrm{Na}]^{+}, 426 \\
{[\mathrm{M}+2 \mathrm{Na}]^{+}, 191\left[\mathrm{C}_{12} \mathrm{H}_{15} \mathrm{O}_{2}\right]^{+}}\end{array}$ & $379[\mathrm{M}-\mathrm{H}]^{-}, 411$ & 284 & Riligustilide & A \\
\hline 53 & 14.70 & $\begin{array}{l}381[\mathrm{M}+\mathrm{H}]^{+}, 403[\mathrm{M}+\mathrm{Na}]^{+}, 191 \\
{\left[\mathrm{C}_{12} \mathrm{H}_{15} \mathrm{O}_{2}\right]^{+}}\end{array}$ & - & 280 & Tokinolide B & A \\
\hline 54 & 12.60 & $\begin{array}{l}381\left[\mathrm{M}+\mathrm{H}^{+}, 399\left[\mathrm{M}+\mathrm{H}_{2} \mathrm{O}+\mathrm{H}^{+}\right.\right. \\
191\left[\mathrm{C}_{12} \mathrm{H}_{15} \mathrm{O}_{2}\right]^{+}\end{array}$ & $379[\mathrm{M}-\mathrm{H}]^{-}, 397\left[\mathrm{M}-\mathrm{H}+\mathrm{H}_{2} \mathrm{O}\right]^{-}$ & 230,276 & Levistolide A & $A, B, C, D, E$ \\
\hline 55 & 3.01 & $\begin{array}{l}613[\mathrm{M}+\mathrm{H}]^{+}, 635[\mathrm{M}+\mathrm{Na}]^{+}, 451 \\
433,163,144\end{array}$ & $611[\mathrm{M}-\mathrm{H}]^{-}, 543,353,191$ & 227 & Hydroxysafflor yellow A & B \\
\hline 56 & 4.01 & $\begin{array}{l}625[\mathrm{M}+\mathrm{H}]^{+}, 593,481,433,319 \\
301,197\end{array}$ & $623[\mathrm{M}-\mathrm{H}]^{-}, 611,525,479,395$ & 232 & $\begin{array}{l}\text { Isorhamnetin-3-O- } \\
\text { nehesperridin }\end{array}$ & B \\
\hline 57 & 2.56 & $803[\mathrm{M}+\mathrm{H}]^{+}, 789,627,325$ & $801[\mathrm{M}-\mathrm{H}]^{-}, 787,593,515,236$ & $230,269,328$ & $\begin{array}{l}\text { 6-hydroxy-keampferol -3, } \\
\text { 6-O-7-O-glucuronide }\end{array}$ & B \\
\hline 58 & 3.85 & $627[\mathrm{M}+\mathrm{H}]^{+}, 481,319,197$ & $625[\mathrm{M}-\mathrm{H}]^{-}, 525,479,449$ & 232 & $\begin{array}{l}\text { 6- hydroxy-keampferol } \\
-3,6-0 \text { - glucoside }\end{array}$ & B \\
\hline 59 & 5.13 & $\begin{array}{l}595[\mathrm{M}+\mathrm{H}]^{+}, 617[\mathrm{M}+\mathrm{Na}]^{+}, 449 \\
287\end{array}$ & 593[M-H]', 515, 449, 341, 193 & $241,265,330$ & Safflor yellow A & B \\
\hline 60 & 7.29 & $303[\mathrm{M}+\mathrm{H}]^{+}$ & $301[\mathrm{M}-\mathrm{H}]^{-}$ & 275 & Queretin & $\mathrm{D}$ \\
\hline 61 & 4.81 & $625[\mathrm{M}+\mathrm{H}]^{+}, 317,479$ & $623[\mathrm{M}-\mathrm{H}]^{-}, 525,315$ & 253,351 & $\begin{array}{l}\text { Isohammetin-3-O- } \\
\text { neohesperidoside }\end{array}$ & $\mathrm{D}$ \\
\hline 62 & 5.25 & $625[\mathrm{M}+\mathrm{H}]^{+}, 370,356,325,317$ & $623[\mathrm{M}-\mathrm{H}]^{-}, 515,315,309,279,181$ & 244,332 & $\begin{array}{l}\text { Isohammetin-3-O- } \\
\text { rutinoside }\end{array}$ & $\mathrm{D}$ \\
\hline
\end{tabular}


Table 2 Characterization of compounds in Siwu decoction categorized formulae by UPLC-QTOF-MS/MS (Continued)

\begin{tabular}{|c|c|c|c|c|c|c|}
\hline 63 & 4.40 & $\begin{array}{l}771[\mathrm{M}+\mathrm{H}]^{+}, 793[\mathrm{M}+\mathrm{Na}]^{+}, 625 \\
{[\mathrm{M}+\mathrm{H}-\mathrm{rha}]^{+}, 479[\mathrm{M}+\mathrm{H}-2 \mathrm{rha}]^{+}} \\
317[\mathrm{M}+\mathrm{H}-3 \mathrm{rha}]^{+}\end{array}$ & $769[\mathrm{M}-\mathrm{H}]^{-}, 525,449,327$ & 253,353 & Typhaneoside & $\mathrm{D}$ \\
\hline 64 & 12.10 & $287[\mathrm{M}+\mathrm{H}]^{+}$ & $285[\mathrm{M}-\mathrm{H}]^{-}$ & 279 & Keampferol & $\mathrm{D}$ \\
\hline 65 & 5.64 & $317[\mathrm{M}+\mathrm{H}]^{+}$ & $315[\mathrm{M}-\mathrm{H}]^{-}$ & 230,273 & Isohamnetin & $\mathrm{D}$ \\
\hline 66 & 4.90 & $463[\mathrm{M}+\mathrm{H}]^{+}, 352,322$ & $461[\mathrm{M}-\mathrm{H}]^{-}, 285,193$ & 235,323 & Scutellarin & $\mathrm{E}$ \\
\hline 67 & 6.33 & $447[\mathrm{M}+\mathrm{H}]^{+}, 336,352,271$ & $445[\mathrm{M}-\mathrm{H}], ; 891,269$ & $216,277,316$ & Baicalin & E \\
\hline 68 & 7.48 & $461[\mathrm{M}+\mathrm{H}]^{+}, 285$ & $459[\mathrm{M}-\mathrm{H}]$, 919, 283 & 220,273 & Wogonoside & E \\
\hline 69 & 8.83 & $271[\mathrm{M}+\mathrm{H}]^{+}$ & $269[\mathrm{M}-\mathrm{H}]^{-}$ & 275,322 & Baicalein & E \\
\hline 70 & 10.46 & $285[\mathrm{M}+\mathrm{H}]^{+}$ & $283[\mathrm{M}-\mathrm{H}]^{-}, 268\left[\mathrm{M}-\mathrm{H}-\mathrm{CH}_{3}\right]^{-}$ & 274 & Wogonin & E \\
\hline 71 & 7.19 & $461[\mathrm{M}+\mathrm{H}]^{+}, 285$ & $459[\mathrm{M}-\mathrm{H}]]^{-}, 283,175$ & 271,310 & Oroxylin-A-glucuroside & E \\
\hline 72 & 10.66 & $315,373,283$ & $313[\mathrm{M}-\mathrm{H}]]^{-}, 375,285$ & 271 & 6-dimethoxy-wogonin & E \\
\hline 73 & 0.64 & $136[\mathrm{M}+\mathrm{H}]^{+}$ & $134[\mathrm{M}-\mathrm{H}]^{-}$ & & Tetramethylpyrazine & $A, B, C, D, E$ \\
\hline 74 & 5.36 & $\begin{array}{l}356\left[\mathrm{M}+\mathrm{H}^{+}, 338\left[\mathrm{M}+\mathrm{H}-\mathrm{H}_{2} \mathrm{O}\right]^{+}\right. \\
312\left[\mathrm{M}+\mathrm{H}-\mathrm{CO}_{2}\right]^{+}\end{array}$ & $354[\mathrm{M}-\mathrm{H}]^{-}$ & 239,297 & Tetrahydropalmatin & $C, D, E$ \\
\hline 75 & 4.56 & $342[\mathrm{M}+\mathrm{H}]^{+}, 311,193,179$ & - & 237,278 & Tetrahydrocolumbamine & $C, D$ \\
\hline 76 & 5.71 & $\begin{array}{l}354[\mathrm{M}+\mathrm{H}]^{+}, 376[\mathrm{M}+\mathrm{Na}]^{+}, 340 \\
320\end{array}$ & $352[\mathrm{M}-\mathrm{H}]^{-}$ & 238,285 & Protopine & $C, D$ \\
\hline 77 & 5.26 & $\begin{array}{l}370\left[\mathrm{M}+\mathrm{H}^{+}, 356\left[\mathrm{M}+\mathrm{H}-\mathrm{CH}_{2}\right]^{+}\right. \\
327\left[\mathrm{M}+\mathrm{H}-\mathrm{CH}_{3}-\mathrm{CO}\right]^{+}, 326[\mathrm{M}+\mathrm{H}- \\
\left.\mathrm{CO}_{2}\right]^{+}\end{array}$ & $368[\mathrm{M}-\mathrm{H}]^{-}$ & 245,330 & Allocryptopine & $C, D, E$ \\
\hline 78 & 5.40 & $324[\mathrm{M}+\mathrm{H}]^{+}, 338,356$ & $322[\mathrm{M}-\mathrm{H}]^{-}$ & - & Tetrahydrocoptisine & $C, D, E$ \\
\hline 79 & 6.29 & $356[\mathrm{M}+\mathrm{H}]^{+}, 207$ & $354[\mathrm{M}-\mathrm{H}]^{-}$ & - & Glaucine & $C, D, E$ \\
\hline 80 & 6.22 & $336[\mathrm{M}+\mathrm{H}]^{+}, 207$ & $354[\mathrm{M}-\mathrm{H}]^{-}$ & 277 & Berberine & $C, D, E$ \\
\hline 81 & 6.48 & $366[\mathrm{M}+\mathrm{H}]^{+}, 308$ & - & 262,332 & Dehydrocorydaline & $C, D, E$ \\
\hline 82 & 5.78 & $368[\mathrm{M}+\mathrm{H}]^{+}, 352\left[\mathrm{M}+\mathrm{H}-\mathrm{H}_{2} \mathrm{O}\right]^{+}$ & $366[\mathrm{M}-\mathrm{H}]^{-}$ & $239,277,331$ & Corydaline & $C, D, E$ \\
\hline 83 & 6.52 & $\begin{array}{l}339\left[\mathrm{M}+\mathrm{H}^{+}, 357\left[\mathrm{M}+\mathrm{H}+\mathrm{H}_{2} \mathrm{O}\right]^{+}\right. \\
320\left[\mathrm{M}-\mathrm{H}_{2} \mathrm{O}\right]\end{array}$ & $337[\mathrm{M}-\mathrm{H}]^{-}$ & 237,285 & Jatrorrhizine & $C, E$ \\
\hline 84 & 1.44 & $127[\mathrm{M}+\mathrm{H}]^{+}, 149[\mathrm{M}+\mathrm{Na}]^{+}$ & 125[M-H]', $169\left[\mathrm{M}-\mathrm{H}+\mathrm{HCOO}^{-}\right.$ & 215,272 & $\begin{array}{l}\text { 5-hydroxymethyl-2- } \\
\text { furfural (5-HMF) }\end{array}$ & A \\
\hline
\end{tabular}


Figure 2 The intensity trend plots of sixteen representative changed common components in SWDCF samples. $X$ axis represent for SWDCF samples (XF: XFSWD; TH: THSWD; SW: SWD; QL: QLSWD; SF: SFZYD). Y axis represent for relative quantity of target compounds.

group, benzoyl group, which lead to the occurrence of ions at $\mathrm{m} / \mathrm{z} 185,219$ or 121 . Based on fragmentation patterns, the compounds 14,16 , and 18 were identified albiflorin, paeoniflorin, and isopaeoniflorin with MS characteristics of $m / z 481[\mathrm{M}+\mathrm{H}]^{+}, m / z 503[\mathrm{M}+\mathrm{Na}]^{+}$, and $m / z$ 481 $[\mathrm{M}+\mathrm{H}]^{+}$by comparing to reference compounds. And compound $\mathbf{1 8}$ had a typical ion of $\mathrm{m} / \mathrm{z}$ $525\left[\mathrm{M}+\mathrm{HCOO}^{-}\right.$at $\mathrm{ES}^{-}$mode.

Structures of compounds 17, 19, 22, 24 and 25 were deduced from their characteristic UV and MS spectra and fragmentation patterns. According to the literature [18-20], compounds 17, 19 and 22 were tentatively identified as oxypaeoniflorin, galloylpaeoniflorin and mudanpioside I, respectively. The fragmentation patterns of compounds $\mathbf{2 4}$ and 25 were similar with those of monoterpene glycosides. In the ESI-MS/MS experiment, the diagnostic ions of $m / z$ $585[\mathrm{M}+\mathrm{H}]+, m / z 463[\mathrm{M}+\mathrm{H}$ - benzoyl $]+, m / z 179[\mathrm{M}+$ $\mathrm{H}$ - benzoyl - glu],$+ m / z 151$ [M + H - benzoyl - glu -CO] + at, and $m / z 629[\mathrm{M}+\mathrm{HCOO}]-$ at ES- mode. According to the literature [17], the compound 24 ( $\mathrm{tR}=7.90 \mathrm{~min}), 25$ $(\mathrm{tR}=8.09 \mathrm{~min}$ ) were tentatively identified as benzoylalbiflorin, and isobenzoylpaeoniflorin, respectively.

Compounds 13, 15, 23, and 28 were identified as sulfonates of monoterpene glycosides. Compound $\mathbf{1 3}$ was $\mathrm{C}_{23} \mathrm{H}_{28} \mathrm{O}_{13} \mathrm{~S}$ and had ions of $m / z 495\left[\mathrm{M}-\mathrm{CH}_{2} \mathrm{OH}-\mathrm{H}_{2} \mathrm{O}\right]^{-}$, $461\left[\mathrm{M}-\mathrm{SO}_{2}-\mathrm{H}_{2} \mathrm{O}-\mathrm{H}\right]^{-}, 341\left[\mathrm{M}-\mathrm{SO}_{2}-\mathrm{C}_{7} \mathrm{H}_{5} \mathrm{O}-2 \mathrm{OH}\right]^{-}$and 243 [M-glu- benzoyl- $\left.\mathrm{H}_{2} \mathrm{O}-\mathrm{CH}_{3}\right]^{-}$. Compound 13 was plausibly identified as paeoniflorin sulfonate [21]. With the similar MS characteristics, compounds 15, 23, 28 were tentatively identified as galloylpaeoniflorin sulfonate, benzoypaeoniflorin sulfonate, isomaltopaeoniflorin sulfonate [18-20].

Compounds 11, 12, 20, 27 and 30 all exhibited glucosyl group. According to the MS fragments and $\mathrm{UV}_{\lambda \max }$ documented in the literature [22], compounds $11,12,20,27$ and 30 were tentatively identified as $1-O-\beta-$ D- glucopyranosyl -paeonisuffrone, $1^{\prime}$-O -galloylsucrose, pentagalloylglucose, isomaltopaeoniflorin or $6^{\prime}-\mathrm{O}-\beta-\mathrm{D}-$ glucopyranosylalbiflorin, and 6-O- $\beta$-D-glucopyranosyl lactinolide, respectively.

Three phenylpropanoid glycosides were detected and tentatively assigned as acteoside/isoacteoside / forsythoside A (21), cistanoside A or jionoside A1/A2 (26), decaffeoylverbascoside (29) according to the literatures [21,23-25]. The MS spectrums were listed in Table 2.

The Rehmanniae Radix is rich sources of iridoid glycosides [21,23-25]. At the positive and negative MS experiments, 9 iridoid compounds were analyzed and

Figure 2 (See legend on next page.) 

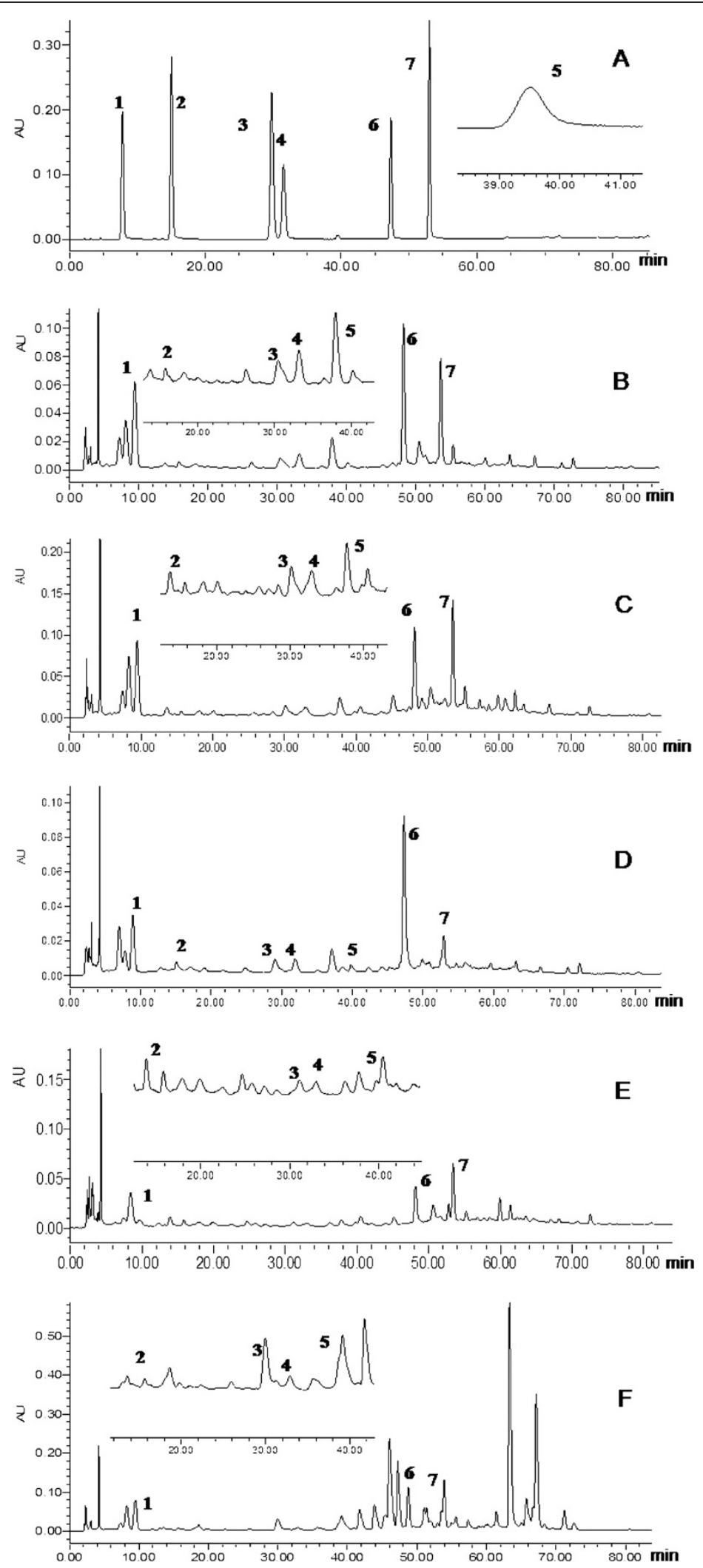

Figure 3 Typical HPLC-DAD chromatograms of mixed standards and samples. A: mixed standards; B: SWD; C: THSWD; D: XFSWD; E: SFZYD; F: QLSWD (1. gallic acid, 2. protocatechuic acid,3. vanillic acid, 4. caffeic acid, 5. paeoniflorin, 6. ferulic acid, 7. senkyunolide I). 
Table 3 Calibration curves, LOD and LOQ data of investigated compounds by HPLC-DAD

\begin{tabular}{|c|c|c|c|c|c|}
\hline Analytes & Calibration curves $^{a}$ & $r^{2}$ & $\begin{array}{c}\text { Linear range } \\
(\mu \mathrm{g} / \mathrm{mL})\end{array}$ & $\begin{array}{c}\text { LOD } \\
(\mu \mathrm{g} / \mathrm{mL})\end{array}$ & $\begin{array}{c}\mathrm{LOQ} \\
(\mu \mathrm{g} / \mathrm{mL})\end{array}$ \\
\hline Gallic acid & $Y=2.4 \times 10^{6} X-8022.9$ & 0.9999 & $148.0-1480.0$ & 7.5 & 14.1 \\
\hline Protocatechuic acid & $Y=8.0 \times 10^{6} X-36350$ & 0.9994 & $38.4-384.0$ & 8.6 & 19.2 \\
\hline Vanillic acid & $Y=2.6 \times 10^{6} X-18490$ & 0.9997 & $32.0-320.0$ & 5.0 & 10.0 \\
\hline Caffeic acid & $Y=3.5 \times 10^{6} X-25506$ & 1.0000 & $35.6-356.0$ & 6.5 & 13.6 \\
\hline Paeoniflorin & $Y=1.1 \times 10^{6} X+160000$ & 0.9990 & $476.0-4760.0$ & 24.8 & 59.6 \\
\hline Ferulic acid & $Y=1.1 \times 10^{7} X-1.2 \times 10^{5}$ & 0.9997 & $136.0-1360.0$ & 6.2 & 12.4 \\
\hline Senkyunolide I & $Y=6.5 \times 10^{6} X-9533.5$ & 0.9999 & $46.0-460.0$ & 6.3 & 12.6 \\
\hline
\end{tabular}

${ }^{a} y$ is the logarithmic value of peak area and $x$ is the logarithmic value of the reference compound's concentration $(\mu \mathrm{g} / \mathrm{ml})$.

identified in SWDCF expcept SFZYD (Table 2). By comparing the $\mathrm{t} R, \mathrm{UV}_{\lambda \max }$ and MS characteristics with reference compounds and literature [23,26], they were identified as rehmannioside D (31), melittoside (32), echinacoside (33), rehmaionoside A/B (34), jionoside B1/B2 (35), martynoside isomer (36), martynoside (37), rehmapicroside (38), and rehmapicrogenin (39), respectively. And amygdalin (40) was detected and identified in THSWD.

\section{Identification of lactones}

Angelicae sinensis Radix and Chuanxiong Rhizoma are rich sources of lactones or phthalide compounds [16,26-28]. In the positive ion mode, 14 lactones were analyzed and identified by comparing with reference compounds and literature data $[27,29]$. Compounds 41, 42, and 43 were tentatively identified as senkyunolide J (41), senkyunolide I (42), and senkyunolide H (43), respectively.

Compounds 44, 45, 49 and 50 were isomers of ligustilide and butylideniphthalide. The $Z$-Ligustilide and $Z$-Butylidenephthalide were adopted as references, the compounds 44 and $\mathbf{4 5}$ were isomers and identified as $E$ ligustilide and $Z$ - ligustilide with MS ion $m / z 191[\mathrm{M}+$ $\mathrm{H}]^{+}$; the compounds $\mathbf{4 9}$ and $\mathbf{5 0}$ were isomers and identified as $E$-Butylideniphthalide and $Z$-Butylidenephthalide with MS ion of $m / z 189[\mathrm{M}+\mathrm{H}]^{+}$, respectively [22].
Six dimmer compounds were identified from SWDCF possessing the same MS fragments ion $m / z 381[\mathrm{M}+\mathrm{H}]^{+}$. According to the literature data $[21,28,30]$, these compounds were tentatively identified as $Z$-ligustilide dimmer E-232 (46), $Z, Z^{\prime}-3,3^{\prime}, 8,8^{\prime}$-Diligustilide (47), angelicide (51), riligustilide (52), tokinolide B (53), and levistolide A (54), respectively.

\section{Identification of flavonoids}

Among 18 flavonoids identified from SWDCF, there were eight flavonoid aglycones including quercetin (60), isorhamnetin (65), keampferol (64), scutellarin (66), baicalein (69), wogonin (70), 6-dimethoxy-wogonin (72), and oroxylinA (71). The flavonoid glycosides were identified

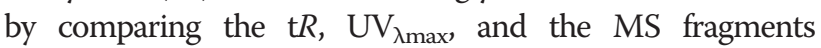
characteristics to the standard substances.

Compounds 61, 62, and $\mathbf{6 3}$ contained fragment ion $\mathrm{m} / \mathrm{z}$ 317 (Table 2). The MS/MS of $\mathrm{m} / z$ showed fragments including $m / z 287,273,153$, and 123 . These data were consistent with those in the literatures [31,32]. The aglucone was identified as isohamnetin. The three flavonoid glycosides were identified as isohamnetin-3-O-neohesperidoside (61), isohamnetin-3-O-rutinoside (62), and typhaneoside (63), respectively.

Hydroxysafflor yellow A (55) and safflor yellow A (59) were detected in THSWD from Carthami Flos. Compound 59 possessed MS ions characteristics of $\mathrm{m} / \mathrm{z} 595$

Table 4 Precision, repeatability of seven analytes

\begin{tabular}{|c|c|c|c|c|c|c|c|}
\hline \multirow[t]{2}{*}{ Analytes } & \multicolumn{2}{|c|}{ Precision (RSD,\%) } & \multicolumn{5}{|c|}{ Repeatability (RSD,\%; $n=6)$} \\
\hline & $\begin{array}{c}\text { Intraday } \\
(n=6)\end{array}$ & $\begin{array}{c}\text { Interday } \\
(n=6)\end{array}$ & SWD & SFZYD & XFSWD & THSWD & QLSWD \\
\hline Gallic acid & 1.75 & 1.78 & 1.81 & 1.26 & 2.84 & 3.32 & 1.31 \\
\hline Protocatechuic acid & 0.76 & 1.21 & 3.16 & 1.90 & 2.72 & 4.18 & 3.19 \\
\hline Vanillic acid & 1.31 & 1.55 & 1.79 & 1.05 & 2.26 & 3.97 & 2.80 \\
\hline Caffeic acid & 0.98 & 0.97 & 2.27 & 2.47 & 1.60 & 4.52 & 3.04 \\
\hline Paeoniflorin & 1.06 & 2.01 & 2.72 & 3.58 & 2.38 & 3.96 & 2.19 \\
\hline Ferulic acid & 0.88 & 1.05 & 2.19 & 3.01 & 1.26 & 2.95 & 2.22 \\
\hline Senkyunolide I & 1.16 & 1.12 & 2.22 & 3.97 & 1.43 & 1.82 & 3.75 \\
\hline
\end{tabular}


Table 5 Stability and recovery of seven analytes

\begin{tabular}{|c|c|c|c|c|c|c|c|c|c|c|c|c|c|c|c|}
\hline \multirow[t]{3}{*}{ Analytes } & \multicolumn{5}{|c|}{ Stability (RSD,\%; $n=6)$} & \multicolumn{10}{|c|}{ Recovery $(\% ; n=6)$} \\
\hline & \multirow[t]{2}{*}{ A } & \multirow[t]{2}{*}{ B } & \multirow[t]{2}{*}{ C } & \multirow[t]{2}{*}{ D } & \multirow[t]{2}{*}{$\mathrm{E}$} & \multicolumn{2}{|c|}{ SWD } & \multicolumn{2}{|c|}{ THSWD } & \multicolumn{2}{|c|}{ XFSWD } & \multicolumn{2}{|c|}{ SFZYD } & \multicolumn{2}{|c|}{ QLSWD } \\
\hline & & & & & & Mean & $\begin{array}{l}\text { RSD } \\
\text { (\%) }\end{array}$ & Mean & $\begin{array}{l}\text { RSD } \\
\text { (\%) }\end{array}$ & Mean & $\begin{array}{l}\text { RSD } \\
\text { (\%) }\end{array}$ & Mean & $\begin{array}{l}\text { RSD } \\
\text { (\%) }\end{array}$ & Mean & $\begin{array}{l}\text { RSD } \\
(\%)\end{array}$ \\
\hline Gallic acid & 6.36 & 3.34 & 7.13 & 5.16 & 1.03 & 98.1 & 2.71 & 98.9 & 1.32 & 97.8 & 2.32 & 101.2 & 3.12 & 99.7 & 1.44 \\
\hline Protocatechuic acid & 5.79 & 5.46 & 6.18 & 4.0 & 4.62 & 101.2 & 3.32 & 99.8 & 1.43 & 97.6 & 1.18 & 103.7 & 3.54 & 102.7 & 1.98 \\
\hline Vanillic acid & 3.14 & 3.68 & 6.25 & 8.80 & 2.19 & 96.2 & 1.39 & 97.3 & 1.79 & 102.3 & 1.25 & 92.2 & 2.81 & 103.1 & 2.32 \\
\hline Caffeic acid & 6.61 & 5.10 & 8.50 & 2.47 & 7.87 & 103.8 & 1.48 & 95.8 & 3.24 & 98.3 & 2.50 & 98.6 & 2.44 & 98.2 & 3.16 \\
\hline Paeoniflorin & 6.13 & 3.79 & 7.37 & 8.36 & 4.07 & 97.1 & 3.25 & 98.2 & 2.77 & 95.4 & 1.15 & 94.0 & 1.36 & 95.8 & 2.17 \\
\hline Ferulic acid & 4.54 & 3.74 & 1.31 & 7.65 & 2.10 & 99.7 & 1.22 & 103.2 & 1.94 & 99.3 & 1.43 & 97.5 & 2.65 & 104.6 & 1.89 \\
\hline Senkyunolide I & 6.87 & 2.82 & 2.47 & 8.91 & 4.07 & 96.9 & 2.31 & 98.1 & 2.13 & 98.1 & 2.86 & 94.0 & 1.91 & 98.1 & 2.34 \\
\hline
\end{tabular}

$[\mathrm{M}+\mathrm{H}]^{+}$and $m / z 617[\mathrm{M}+\mathrm{Na}]^{+}$at $\mathrm{ES}^{+}$mode and $m / z$ $593[\mathrm{M}-\mathrm{H}]^{-}$at $\mathrm{ES}^{-}$mode. Compound 55 had diagnostic ions of $m / z 613[\mathrm{M}+\mathrm{H}]^{+}$, and $m / z 635[\mathrm{M}+\mathrm{Na}]^{+}$at $\mathrm{ES}^{+}$ mode and $m / z 611[\mathrm{M}-\mathrm{H}]^{-}$at $\mathrm{ES}^{-}$mode. Baicalin $(67)$ and wogonoside (68) were identified from QLSWD [33,34].

\section{Identification of alkaloids}

The alkaloids compounds derived mainly from Chuanxiong Rhizoma, Corydalis Rhizoma, and Coptidis Rhizoma. At the positive ion mode, $\mathbf{1 1}$ alkaloids constituents were analyzed and identified from SWDCF by comparing with reference compounds and literature data [35]. Compound 74 with MS characteristics of $m / z 356[\mathrm{M}+\mathrm{H}]^{+}, 338\left[\mathrm{M}+\mathrm{H}-\mathrm{H}_{2} \mathrm{O}\right]^{+}$, and $312\left[\mathrm{M}+\mathrm{H}-\mathrm{CO}_{2}\right]^{+}$was identified as tetrahydropalmatine compared with the reference standard. Compounds 73, 76, $78,80,81,82$, and 83 were detected and identified from XFSWD, SFZYD, and QLSWD as tetramethylpyrazine (73), protopine (76), tetrahydrocoptisine (78), berberine $(\mathbf{8 0})$, dehydrocorydaline (81), corydaline (82), and jatrorrhizine (83) by comparing with the reference standards, respectively. The MS characteristics were $m / z 136[\mathrm{M}+\mathrm{H}]^{+}, m / z 354$ $[\mathrm{M}+\mathrm{H}]^{+}, m / z 324[\mathrm{M}+\mathrm{H}]^{+}, m / z 336[\mathrm{M}+\mathrm{H}]^{+}, m / z 366$ $[\mathrm{M}+\mathrm{H}]^{+}, m / z 368[\mathrm{M}+\mathrm{H}]^{+}$, and $m / z 339[\mathrm{M}+\mathrm{H}]^{+}$, respectively. According to the literature [35], compounds 75, 77,

Table 6 Contents of seven investigated compounds in SWDCF

\begin{tabular}{cccccc}
\hline Analytes & \multicolumn{6}{c}{ Contents of analyst (mean \pm SD; $\mathbf{n = 3 ;} \mathbf{\mu g} / \mathbf{g}$ ) } \\
\cline { 2 - 6 } & SWD & THSWD & XFSWD & SFZYD & QLSWD \\
\hline Gallic acid & 316.00 & 421.20 & 143.50 & 436.00 & 729.80 \\
Protocatechuic acid & 6.50 & 11.60 & 5.40 & 2.46 & 4.05 \\
Vanillic acid & 18.40 & 33.90 & 13.80 & 90.70 & 44.00 \\
Caffeic acid & 58.20 & 69.70 & 31.30 & 67.30 & 84.70 \\
Paeoniflorin & 2050.00 & 3430.00 & 790.00 & 3840.00 & 5140.00 \\
Ferulic acid & 120.00 & 166.70 & 81.30 & 225.70 & 310.70 \\
Senkyunolide I & 180.00 & 30.00 & 70.00 & 210.00 & 80.00 \\
\hline
\end{tabular}

and 79 were tentatively identified as tetrahydrocolumbamine, allocryptopine, and glaucine, respectively. The MS characteristics ions were $m / z 342[\mathrm{M}+\mathrm{H}]^{+}, m / z 370$ $[\mathrm{M}+\mathrm{H}]^{+}$, and $m / z 356[\mathrm{M}+\mathrm{H}]^{+}$, respectively. In addition, 5hydroxymethyl-2-furfural (5-HMF) (84) was identified from SWD only.

UPLC-Q-TOF-MS method was employed to identify the constituents from SWDCF. In ESI-TOF-MS experiment, accurate molecular mass of the components can be obtained. As ESI was a soft ionization technique, the interface produces little fragmentation of analytes and generally forms protonated molecular ions $[\mathrm{M}+\mathrm{H}]^{+}$for positive ionization mode or $[\mathrm{M}-\mathrm{H}]^{-}$for negative ionization mode. Comparing the mass spectra of the compounds with the standards and those in the literature, the common and different components were unequivocally identified from every formula of SWDCF. These data would be provided the bioactive components for activities of different formulae.

\section{The markers obtained by MarkerLynx ${ }^{\mathrm{TM}}$}

The principal components analysis (PCA) was done by the Waters MarkerLynx ${ }^{\text {TM }}$ software. In this study, SWD, THSWD, XFSWD, SFZYD and QLSWD were injected six times each in two ESI modes. Unsupervised PCA was performed to globally evaluate the chemical consistency among these five SWDCF decoctions. The data from both positive and negative ion modes were displayed as scores plots (Additional file 1: Figures S2-1 and 2-2). The scores plots demonstrated a clear classification trend among SWDCF samples, with all the observations falling within the Hotelling T2 (0.95) ellipse, which confirmed the fact that chemical difference exists among SWDCF. The results showed that the SFZYD and QLSWD were significantly different to SWD, THSWD, and XFSWD, while SWD, THSWD, and XFSWD were close to each other. These data indicated that the chemical composition and quantity of components changed after combining SWD with different herbs. 
Chemical markers were analyzed to find out the changed common components contributing most to the SWDCF. Figures 2 showed the representative mass spectra of sixteen common and the greatest change compounds. According to the intensity trends of fragment ions from five decoctions samples, the intensity of the common chemical markers (paeoniflorin 16, albiflorin 14, ferulic acid 5, isoferulic acid 6, senkyunolide I 42, 3-butylidene-7hydroxyphthalide $\mathbf{4 8}$, levistolide A 54, tetramethylpyrazine 73, E-Butylideniphthalide 49, Z-Butylidenephthalide 50, Zligustilide 45, $E$-ligustilide 44, $Z$-ligustilide dimmer 46, gallic acid 1, chlorogenic acid 2, benzoylpaeoniflorin 24) were different in every formula of SWDCF.

The peak areas of components 16 ( $t R 5.80 \mathrm{~min}, \mathrm{~m} / \mathrm{z}$ 481.1750), 14 ( $\mathrm{t} R 3.90 \mathrm{~min}, \mathrm{~m} / z$ 481.1710), 42 ( $\mathrm{t} R 6.26 \mathrm{~min}$, $\mathrm{m} / z$ 225.1144), 54 ( $\mathrm{t} R 12.60 \mathrm{~min}, \mathrm{~m} / \mathrm{z}$ 399.2193), 73 ( $\mathrm{t} R 0.64 \mathrm{~min}, \mathrm{~m} / z$ 136.0622), 49 ( $\mathrm{t} R 8.08 \mathrm{~min}, \mathrm{~m} / z$ 189.0919), 50 ( $\mathrm{t} R 9.27 \mathrm{~min}, \mathrm{~m} / \mathrm{z}$ 189.0919), 46 ( $\mathrm{t} R 13.80 \mathrm{~min}, \mathrm{~m} / \mathrm{z}$ 191.1049), 45 ( $\mathrm{t} R 10.82 \mathrm{~min}, \mathrm{~m} / z$ 191.1079), 44 ( $\mathrm{t} R 9.44 \mathrm{~min}$, $\mathrm{m} / z$ 191.1073) were higher in SWD than other formulae. XFSWD possessed greater peak area of compound 2 and 5, while SFZYD possessed high content of compound 6 .

\section{Optimization of the HPLC conditions}

A small amount of acid was added into the mobile phase which could inhibit the ionization of these components to improve the peak shape and restrain the peak tailing due to the existence of acidic ingredients in SWDCF samples. $0 \%, 0.1 \%$ and $0.2 \%$ aqueous formic acid and acetic acid solutions were compared. The results showed that 7 compounds could be baseline separated when $0.1 \%$ aqueous formic acid solution was selected.

DAD detection was set at the wavelength range of 190-400 nm. For the satisfactory sensitivity, resolution and lower noise, four wavelengths at $230 \mathrm{~nm}, 260 \mathrm{~nm}$, $277 \mathrm{~nm}$, and $320 \mathrm{~nm}$ were selected for determining the different compounds in SWDCF. According to absorption curve of the tested analytes, the paeoniflorin had optimal sensitivity for detection at $230 \mathrm{~nm}, 320 \mathrm{~nm}$ for ferulic acid, $260 \mathrm{~nm}$ for gallic acid, vanillic acid, and caffeic acid, and $277 \mathrm{~nm}$ for senkyunolide I. Thus, a switching UV wavelength method was established by a variable-wavelength spectrophotometric detector. Under the optimized HPLC-UV conditions, the investigated analytes were well separated and detected in $85 \mathrm{~min}$ (Figure 3).

Prior to sample analysis the optimal process of extraction had to be investigated. According to the applied form of decoction, the SWDCF were extracted by refluxing with water for twice. Ethanol (95\%) was added to the filtrates until the concentration of ethanol was adjusted to $50 \%$ and seven compounds were almost completely extracted.

\section{Validation of the quantitative analysis}

The HPLC-DAD method for quantitation analysis was validated to determine the linearity, LOD, and LOQ. Good linear correlation and high sensitivity at these chromatographic conditions were confirmed by the correlation coefficients $\mathrm{r}^{2}>0.9990$ and $P=3.7501 \times 10^{-9}, 1.3503 \times 10^{-7}$, $3.7553 \times 10^{-8}, 0,3.7513 \times 10^{-7}, 3.7553 \times 10^{-8}, 3.7501 \times 10^{-9}$ for gallic acid, protocatechuic acid, vanillic acid, caffeic acid, paeoniflorin, ferulic acid, senkyunolide I, respectively, within the test ranges, and the overall LODs and LOQs were in the range of $5.0-24.8$ and $10.0-59.6 \mu \mathrm{g} / \mathrm{mL}$, respectively (Table 3 ).

As shown in Table 4, the intra- and inter-day precisions, repeatability and stability of the seven analytes were less than $3 \%$. The overall recoveries lay between $92.20 \%$ and $104.60 \%$ with RSD less than $3.54 \%$ for seven components in all samples. These results indicated that the HPLC fingerprint chromatograms had a good repeatability, precision, accuracy, and recovery (Tables 4 and 5) and the developed HPLC-DAD method was a reliable and useful method for assessment of SWDCF.

\section{Sample analysis}

The HPLC-DAD method was then subsequently applied to simultaneously determine the chemical markers including gallic acid, protocatechuic acid, vanillic acid, caffeic acid, paeoniflorin, ferulic acid, senkyunolide I in SWDCF samples. The results (Table 6) showed there were remarkable differences among the contents of the chemical markers analyzed in different samples. Paeoniflorin (5) was found to be a predominant constituent in both of QLSWD and SFZYD, while the lowest contents in SWD except XFSWD, suggesting that the active compounds of paeoniflorin was dissolved increasedly after the SWD combined with other herbs. The contents of senkyunolide I in SWDCF were decreased except SFZYD varing from 31.30 to $84.70 \mathrm{mg} / \mathrm{g}$. The phenolic acids including gallic acid, caffeic acid, ferulic acid, vanillic acid, and protocatechuic acid were increased significantly except in XFSWD.

\section{Conclusion}

The chemical profiles with 84 components in SWDCF, including monoterpene glycosides, acetophenones, galloyl glucoses, even some isomers in the complex system were characterized by UPLC-QTOF-MS/MS.

\section{Additional file}

Additional file 1: Figure S1. Typical BPI chromatogram of SWDCF (A) SWD, (B) THSWD, (C) XFSWD, (D) SFZYD, and (E) QLSWD. Figure S1-1 ESI ${ }^{+}$; Figure S1-2 ESI. Figure S2: PCA model results between SWDCF samples. $\left(A, E S I^{+} ; B, E S I^{-}\right)$. 


\section{Abbreviation}

SWDCF: Siwu decoction categorized formulae; UPLC - QTOF - MS /MS: Ultrahigh performance liquid chromatography coupled with time-of-flight mass spectrometry; CM: Chinese medicine; CF: Categorized formulae; SWD: Siwu decoction; THSWD: Taohong Siwu decoction; PD: Primary dysmenorrheal; XFSWD: Xiangfu Siwu decoction; SFZYD: Shaofu Zhuyu decoction; QLSWD: Qinlian Siwu decoction; ESI: Electrospray ionization.

\section{Competing interest}

The authors declare that they have no competing interest.

\section{Authors' contribution}

JD designed the experiment. SS performed the experiments, analyzed the data and wrote the manuscript. WC, and ES performed the experiments. WZ analyzed the data. YT revised the manuscript. All authors read and approved the final version of the manuscript.

\section{Acknowledgements}

This work was supported by Key Research Project in Basic Science of Jiangsu College and University (No. 11KJA360002; 06KJA36022), National Natural Science Foundation of China (No. 30973885), and 2006 Training Program of Scientific and Technological Innovation Team for "Qinglan Project" of Jiangsu College and University. And this work was supported by Construction Project for Jiangsu Key Laboratory for High Technology Research of TCM Formulae (BM2010576), Construction Project for Jiangsu Engineering Center of Innovative Drug from Blood-conditioning TCM Formulae and a Project Funded by the Priority Academic Program Development of Jiangsu Higher Education Institutions (ysxk-2010).

\section{Author details}

'Jiangsu Key Laboratory for TCM Formulae Research, Nanjing University of Chinese Medicine, Nanjing 210023, PR China. ${ }^{2}$ College of Pharmacy, Jiangsu University, Zhenjiang 212013, PR China.

Received: 29 October 2012 Accepted: 14 February 2013

Published: 1 March 2013

\section{References}

1. Li S: Network Systems underlying traditional Chinese medicine syndrome and herb formula. Curr Bioinformat 2009, 4:188-196.

2. Fan XS, Duan JA, Sun SF, Ding AW, Lu Y, Tang YP: Significance of categor ized formula in modern TCM formula. World Science and Technology / Modernization of Tradit Chin Med Mat Med 2007, 9:17-21.

3. Wang ZJ, Wo SK, Wang L, Lau CBS, Lee VHL, Chow MSS, Zuo Z: Simultaneous quantification of active components in the herbs and products of Si-Wu-Tang by high performance liquid chromatographymass spectrometry. J Pharm Biomed Anal 2009, 50:232-244.

4. Cheng JF, Lu ZY, Su YC, Chiang LC, Wang RY: A traditional Chinese herbal medicine used to treat dysmenorrhoea among Taiwanese women. J Clin Nurs 2008, 17:2588-2595.

5. The State Pharmacopoeia Commission of P.R. China: Pharmacopoeia of the People's Republic of China. Beijing: Chemical Industry Press; 2005.

6. Hua YQ, Duan JA, Su SL, Lu Y, Wang QJ, Tang YP: Bioactivity evaluation of Siwu decoction and its derived formulae in the treatment of primary dysmenorrheal. J Chin Pharmaceut Univ 2008, 39:72-76.

7. Zhang CB, Lu Y, Duan JA, Su SL, Tang YP, Shang EX: Study on Siwu decoction and its serial decoctions in dysmenorrhea model mice. Pharm Clin Res 2007, 15:459-461.

8. Su SL, Hua YQ, Duan JA, Tang YP, Lu Y, Ding AW: In vitro inhibition on the contraction of isolated mouse uterine and chemical components of Shaofu Zhuyu decoction. J Chin Pharmaceut Univer 2007, 38:544-549.

9. Liu L, Duan JA, Tang YP, Ma HY, Su SL, Li XX: Study on antioxidant effect and chemical constituents of Taohong Siwu decoction. Chin J Chin Mater Med 2011, 36:1591-1595.

10. Su SL, Duan JA, Wang TJ, Yu L, Hua YQ, Tang YP: Evaluating the effects of Shaofu Zhuyu decoction on hemorheology and ovarian functionin rat model of Han-Ning blood stasis. Chin J Exp Tradit Med Form 2008, 14:14-16.

11. Liu L, Duan JA, Zhu ZH, Shi XQ, Su SL, Tang YP, Qian DW: Correlation of combinations between angelicae sinensis radix and carthami flos. Chin Tradit Herbal Drugs 2011, 42:929-934.
12. Zhu M, Tang YP, Duan JA, Guo JM, Guo S, Su SL, Shang EX, Qian DW, Ding AW: Roles of paeoniflorin and senkyunolide I in SiWu decoction on antiplatelet and anticoagulation activities. J Sep Sci 2010, 33:3335-3340.

13. Liu CM, Tang YP, Ding AW, WU DK, Duan JA: Simultaneous determination of eight main active compounds in Qin-Lian-Si-Wu decoction by HPLCDAD. Asian Chem Lett 2009, 3:73-79.

14. National Commission of Chinese Pharmacopoeia: Pharmacopoeia of Peoples Republic of China. Chemical Industry Press: Beijing; 2010:386. 26, 60, 127, 89.

15. Su SL, Guo JM, Duan JA, Wang TJ, Qian DW, Shang EX, Tang YP: Ultraperformance liquid chromatography-tandem mass spectrometry analysis of the bioactive components and their metabolites of Shaofu Zhuyu decoction active extract in rat plasma. J Chromatogr B 2010, 878:355-362.

16. Li SL, Song JZ, Qiao CF, Zhou Y, Xu HX: UPLC-PDA-TOFMS based chemical profiling approach to rapidly evaluate chemical consistency between traditional and dispensing granule decoctions of traditional medicine combinatorial formulae. J Pharm Biomed Anal 2010, 52:468-478

17. Huang WY, Sheu SJ: Separation and identification of the organic acids in angelicae radix and ligustici rhizoma by HPLC and CE. J Sep Sci 2006, 29:2616-2624

18. Yang L, Xu SJ, Tian RT, Xie PS, Wang ZT: HPLC fingerprinting of radix paeoniae alba. Acta Pharmacol Sinica 2007, 42:71-74.

19. Liu EH, Qi LW, Li B, Peng YB, Li P, Li CY, Cao J: High-speed separation and characterization of major constituents in radix paeoniae rubra by fast high-performance liquid chromatography coupled with diode-array detection and time-of-flight mass spectrometry. Rapid Commun Mass Spectrom 2009, 23:119-130.

20. Xu SJ, Yang L, Zeng X, Zhang M, Wang ZT: Characterization of compounds in the Chinese herbal drug Mu-Dan-Pi by liquid chromatography coupled to electrospray ionization mass spectrometry. Rapid Commun Mass Spectrom 2006, 20:3275-3288.

21. Cheng YS, Peng C, Zhang H, Wen FY: Structural characterization of an artifact and simultaneous quantification of two monoterpenes and their artifacts of isolation in white-peony root. Helvetica Chimica Acta 2010, 93:565-572.

22. Qi LW, Li P, Li SL, Sheng LH, Li RY, Song Y, Li HJ: Screening and identification of permeable components in a combined prescription of Danggui Buxue decoction using a liposome equilibrium dialysis systemfollowed by HPLC and LC-MS. J Sep Sci 2006, 29:2211-2220.

23. Nishimura H, Sasaki H, Morota T, Masao C, Mitsuhashi H: Six glycosides from Rehmannia glutinosa var. Purpurea. Phytochemistry 1990, 29:3303-3306.

24. Sasaki H, Nishimura H, Chin M, Mitsuhashi H: Hydroxycinnamic acid esters of phenethylalcohol glycosides from Rehmannia glutinosa var. Purpurea. Phytochemistry 1989, 28:875-879.

25. Sasaki H, Nishimura H, Morota T, Chin M, Wei H, Yu-Lang X: Chemical and biological studies on rehmanniae radix. Part 1. Immunosuppressive principles of Rehmannia glutinosa var. hueichingensis. Planta Med 1989, 55:458-462.

26. Zhan XF, Li P, Sun YQ: HPLC/ESI-MS analysis of the radix rehmanniae praeparata. Chin J Pharm Anal 2007, 27:874-876.

27. Lin LZ, He XG, Lian LZ, King W, Elliott J: Liquid chromatographicelectrospray mass spectrometric study of the phthalides of Angelica sinensis and chemical changes of Z-ligustilide. J Chromatogr A 1998, 810:71-79.

28. Lu GH, Chan K, Liang YZ, Leung K, Chan CL, Jiang ZH, Zhao ZZ: Development of high-performance liquid chromatographic fingerprints for distinguishing Chinese Angelica from related umbelliferae herbs. J Chromatogr A 2005, 1073:383-392.

29. Yi T, Leung KSY, Lu GH, Chan K, Zhang H: Simultaneous qualitative and quantitative analyses of the major constituents in the Rhizome of Ligusticum Chuanxiong using HPLC-DAD-MS. Chem Pharm Bull 2006, 54:255-259.

30. Deng SX, Chen SN, Lu J, Wang ZJ, Nikolic D, van Breemen RB, Santarsiero BD, Mesecar A, Fong HH, Farnsworth NR, Pauli GF: GABAergic phthalide dimers from Angelica sinensis (Oliv.)Diels. Phytochem Anal 2006, 17:398-405.

31. Tao WW, Yang NY, Duan JA, Wu DK, Guo JM, Tang YP, Qian DW, Zhu ZH: Simultaneous determination of eleven major flavonoids in the pollen of Typha angustifolia by HPLC-PDA-MS. Phytochem Anal 2011, 22:455-461.

32. Liao MC, Liu YL, Xiao PG: Studies on the flavonoids from the pollens of Typha Davidiana, T. Latifolia and T. angustata (Pu Huang). Acta Bot Sin 1989, 31:939-947. 
33. Li HB, Jiang $Y$, Chen F: Separation methods used for Scutellaria baicalensis active components. J Chromatogr B 2004, 812:277-290.

34. Collado G, Macias FA, Massanet GM, Luis FR: Flavonoid from Centaurea clementei. J Nat Prod 1985, 48:819-822.

35. Zhang J, Jin Y, Dong J, Xiao Y, Feng J, Xue X, Zhang X, Liang X: Systematic screening and characterization of tertiary and quaternary alkaloids from cordalis yanhusuo W.T. Wang using ultra-performance liquid chromatography - quadrupole - time-of-flight mass spectrometry. Talanta 2009, 78:513-522.

doi:10.1186/1749-8546-8-5

Cite this article as: Su et al:: Chemical fingerprinting and quantitative constituent analysis of Siwu decoction categorized formulae by UPLCQTOF/MS/MS

and HPLC-DAD. Chinese Medicine 2013 8:5.

\section{Submit your next manuscript to BioMed Central and take full advantage of:}

- Convenient online submission

- Thorough peer review

- No space constraints or color figure charges

- Immediate publication on acceptance

- Inclusion in PubMed, CAS, Scopus and Google Scholar

- Research which is freely available for redistribution 